\title{
Online Parameter Identification and State of Charge Estimation of Battery Based on Multitimescale Adaptive Double Kalman Filter Algorithm
}

\author{
Wenxian Duan, ${ }^{1}$ Chuanxue Song, ${ }^{1}$ Yuan Chen, ${ }^{2}$ Feng Xiao, ${ }^{1}$ Silun Peng, \\ Yulong Shao, ${ }^{3}$ and Shixin Song $\mathbb{B}^{4}$ \\ ${ }^{1}$ State Key Laboratory of Automotive Simulation and Control, Jilin University, Changchun 130022, China \\ ${ }^{2}$ School of Electrical Engineering and Automation, Hefei University of Technology, Hefei 230009, China \\ ${ }^{3}$ Zhengzhou Yutong Bus Co., Ltd., Zhengzhou 450016, China \\ ${ }^{4}$ School of Mechanical and Aerospace Engineering, Jilin University, Changchun 130022, China
}

Correspondence should be addressed to Shixin Song; songshx202@126.com

Received 15 August 2020; Revised 26 September 2020; Accepted 17 November 2020; Published 4 December 2020

Academic Editor: Baogui Xin

Copyright (c) 2020 Wenxian Duan et al. This is an open access article distributed under the Creative Commons Attribution License, which permits unrestricted use, distribution, and reproduction in any medium, provided the original work is properly cited.

\begin{abstract}
An accurate state of charge (SOC) can provide effective judgment for the BMS, which is conducive for prolonging battery life and protecting the working state of the entire battery pack. In this study, the first-order RC battery model is used as the research object and two parameter identification methods based on the least square method (RLS) are analyzed and discussed in detail. The simulation results show that the model parameters identified under the Federal Urban Driving Schedule (HPPC) condition are not suitable for the Federal Urban Driving Schedule (FUDS) condition. The parameters of the model are not universal through the HPPC condition. A multitimescale prediction model is also proposed to estimate the SOC of the battery. That is, the extended Kalman filter (EKF) is adopted to update the model parameters and the adaptive unscented Kalman filter (AUKF) is used to predict the battery SOC. The experimental results at different temperatures show that the EKF-AUKF method is superior to other methods. The algorithm is simulated and verified under different initial SOC errors. In the whole FUDS operating condition, the RSME of the SOC is within $1 \%$, and that of the voltage is within $0.01 \mathrm{~V}$. It indicates that the proposed algorithm can obtain accurate estimation results and has strong robustness. Moreover, the simulation results after adding noise errors to the current and voltage values reveal that the algorithm can eliminate the sensor accuracy effect to a certain extent.
\end{abstract}

\section{Introduction}

To solve the problems of the increasing global environment and the depletion of renewable energy, governments around the world advocate the new energy vehicles to replace traditional fuel vehicles. In comparison with lead-acid and $\mathrm{NiMH}$ batteries, lithium batteries have the advantages of high energy density, high rated voltage, high power bearing capacity, and high- and low-temperature adaptability; they have been widely used in electric vehicles $[1,2]$. A good monitoring method plays a very important role in the electrical system, which can accurately collect the physical signals monitored in the system and provide security for the system [3]. The battery management system (BMS), which is a key part of electric vehicles, can effectively manage the working state of the power battery pack and provide security for normal driving. An accurate state of charge (SOC) and state of health can not only effectively judge whether it needs an equalization strategy to ensure that the entire battery is in a stable working state, but also avoid the phenomenon of battery overdischarge to ensure the normal service life of the battery [4].

However, in vehicle driving, the internal working state of the battery is a nonlinear electrochemical reaction, and it is easily affected by the external environment temperature and its own cycle life; thus, obtaining an accurate SOC value is 
difficult [5]. At present, most methods for determining battery SOC include ampere-hour integral [6] and opencircuit voltage (OCV) [7]. Although the above methods are simple and feasible and they can obtain an accurate SOC value, they also have defects. For example, the ampere-hour integration method needs to know the accurate initial SOC value in advance, and the OCV method needs the battery to stop charging and discharging for at least two hours. Several neural networks, such as artificial neural networks [8,9], wavelet neural networks [10], and support vector machine [11], are also used to predict battery SOC. With the improvement of the computing power of computer hardware, some deep learning methods have been applied to battery SOC estimation. These methods include the long short-term memory (LSTM) network [12] and gated recurrent unit (GRU) network [13]. Although these AI algorithms do not require an accurate battery model to obtain accurate battery SOC, these methods need numerous charge and discharge experiments under different working conditions. Then, large-scale iterative training is carried out on the deep learning network through the measured experimental data to obtain the accurate SOC value. But the accuracy of model prediction depends on the quality of experimental data.

At present, as the chemical reaction inside the battery is a nonlinear change, some filtering algorithms have strong robustness in the nonlinear system. An adaptive extended Kalman filter (EKF) is used to estimate the SOC of the second-order battery model to allow the algorithm to retain high estimation accuracy in the case of unknown noise [14]. In [5], an adaptive cubature Kalman filter (CKF) algorithm is used to estimate battery SOC. Although accurate SOC values are obtained, the mathematical formula reasoning shows that when the dimension in the state equation is less than 3 , the prediction accuracy of CKF is lower than that of the unscented Kalman filter (UKF). The model parameters are not fixed under different SOCs and temperatures. In order to obtain a more accurate SOC estimation value, the recursive least squares method with forgetting factor (FFRLS) is proposed to update the model parameters online, and the adaptive unscented Kalman filter (AUKF) is used to forecast the battery SOC [15]. In [16], the least squares method is improved during parameter identification, and the online update of parameters and the accurate estimation of SOC are realized. If the inaccurate initial parameter cannot continue to converge, it will affect the SOC estimation accuracy of the entire working condition experiment. Therefore, some double Kalman filter algorithms are proposed. These algorithms include DEKF [17], EKF-UKF [18], EKF-PF [19], DHIF [20], and DPF [21]. The DPF and the improved DAPF algorithms can obtain more accurate SOC estimation values than the Kalman filter algorithm in the case of non-Gaussian noise. However, given that the variance of particle weight can increase with time iteration, particle degradation is common in particle algorithm. In the sampling stage, EKF, UKF, and CKF algorithms are used to calculate the mean and covariance for each particle, and then the mean and variance are used to sample the particles. This kind of algorithm mainly includes EPF [22], CPF [23], and UPF [24]. However, such calculation amount is higher than that of the ordinary Kalman filter algorithm. In [25], a new method is proposed to update the model parameters by using the $\mathrm{H}$-infinity filter and the SOC by using the UKF algorithm. The frequent parameter updating on the same scale not only does not get an accurate SOC estimation, but also increases the calculation amount. In [17], the Kalman filter is used to update the model parameters and estimate the SOC on different timescales. The results show that it not only improves the convergence speed and SOC estimation accuracy at the initial stage of the algorithm but also decreases the calculation amount. Considering the advantage and disadvantage of the abovementioned algorithms, this paper proposes an EKF algorithm to update the model parameters on the macroscale and an AUKF algorithm to update battery SOC on the microscale. A large number of simulation experiments show that this algorithm has high accuracy and robustness.

The detailed chapters of this paper are as follows. Section 2 introduces the first-order RC battery model and two methods for parameter identification. Section 3 discusses the EKF-AUKF algorithm in detail. Section 4 describes the experimental data in detail and identifies the battery model parameters. Section 5 discusses and analyzes the effects of different initial SOC errors and sensor accuracy on the robustness of the algorithm in detail. Section 6 is the conclusion of this paper.

\section{Battery Model and Parameter Identification}

2.1. Battery Model Description. The battery SOC reflects the situation of residual power. In battery operation, when the unit of sampling time is small, the SOC can be obtained by the ampere-hour integration method:

$$
\operatorname{SOC}(t)=\operatorname{SOC}\left(t_{0}\right)-\frac{\int \eta_{i} I_{t} \mathrm{~d} t}{C_{N}}
$$

where SOC $\left(t_{0}\right)$ represents the value of battery SOC at the time $t_{0}$, SOC $(t)$ is the value of battery SOC at the current time $t, \eta$ is to the coulomb efficiency of the battery, $\eta=1, I_{t}$ refers to the current flowing through the battery and load, and $C_{N}$ refers to the rated capacity of the battery.

The complex electrochemical reaction inside the battery can be regarded as a nonlinear system, which can be replaced by a nonlinear system model. On the basis of the internal reaction mechanism of the battery, an electrochemical equation corresponding to the reaction conditions can be established, and a further accurate residual capacity can be obtained. However, the electrochemical equation is complex and too many model parameters need to be identified, which requires a large amount of calculation [26, 27]. Given that the nonlinear system equation of the battery equivalent circuit model is simple and it can obtain highly accurate battery SOC, it has become the research object of many scholars. In $[28,29]$, by comparing SOC estimation results of common several equivalent circuit models, the second-order $\mathrm{RC}$ model is superior to other battery models. Considering the prediction accuracy and the amount of calculation of the model, the first-order RC model is more suitable for the 
nonlinear battery system. As shown in Figure 1, this paper selects the first-order RC battery model as the research object.

On the basis of Kirchhoff's law, the state and observation equations of the first-order equivalent circuit model can be obtained as follows:

$$
\begin{aligned}
& \dot{U}_{p}=-\frac{1}{C_{p} R_{p}} U_{p}+\frac{1}{C_{p}} I_{t}, \\
& U_{t}=U_{\mathrm{OC}}(\operatorname{SOC}(t))-U_{p}-R_{0} I_{t},
\end{aligned}
$$

where $U_{t}$ represents the measured voltage, $U_{P}$ represents the voltage of the RC network in the model, and $I_{t}$ represents the current flowing through the battery. $U_{\mathrm{OC}}$ is the battery OCV, which has a nonlinear relationship with $\operatorname{SOC}(t), R_{0}$ is ohmic internal resistance, $R_{P}$ is polarization internal resistance, $C_{P}$ is polarization capacitance.

In order to apply the proposed algorithm to battery SOC estimation, equations (1) and (2) are discretized as shown in the following equations:

$$
\left[\begin{array}{c}
\mathrm{SOC}_{k} \\
U_{p, k}
\end{array}\right]=\left[\begin{array}{cc}
1 & 0 \\
0 & \Lambda_{k-1}
\end{array}\right]\left[\begin{array}{c}
\mathrm{SOC}_{k-1} \\
U_{p, k-1}
\end{array}\right]+\left[\begin{array}{c}
-\frac{\eta \Delta T}{C_{N}} \\
R_{p}\left(1-\Lambda_{k-1}\right)
\end{array}\right] I_{k-1}+w_{k-1}
$$

$$
U_{k}=U_{\mathrm{OC}}\left(\mathrm{SOC}_{k}\right)-U_{p, k}-R_{0} I_{k}+v_{k} \text {, }
$$

where $\Lambda_{k-1}=e^{-\Delta T / R_{p} C_{p}}, \Delta T$ is the sampling time of the system, and $w_{k-1}$ and $v_{k}$ are the process noise and measurement noise, respectively.

The experimental data indicates that there is a certain nonlinear relationship between the battery OCV and SOC. The relationship is determined by the Octave polynomial:

$$
\begin{aligned}
U_{\mathrm{OC}}= & H_{1} \mathrm{SOC}^{8}+H_{2} \mathrm{SOC}^{7}+H_{3} \mathrm{SOC}^{6}+H_{4} \mathrm{SOC}^{5} \\
& +H_{5} \mathrm{SOC}^{4}+H_{6} \mathrm{SOC}^{3}+H_{7} \mathrm{SOC}^{2}+H_{8} \mathrm{SOC}+H_{9},
\end{aligned}
$$

where $H_{i}(i=1,2, \ldots, 9)$ is the fitting coefficient of the polynomial.

2.2. Parameter Identification Method. Prior to using the equivalent circuit model for estimating the battery SOC and voltage, the model parameters must be identified offline to decrease the influence of uncertain parameters on the estimation effect. At present, the methods for offline parameter identification mainly include the exponential function fitting data method [30], RLS method, FFRLS method [31], genetic algorithm [32], and particle swarm optimization algorithm [33], and improved ant lion optimizer [34]. Two common parameter identification methods are described in detail as follows.

Figure 2 shows the Simulink simulation environment of model parameter identification. The algorithm is mainly the ordinary RLS. Figure 3 illustrates a battery simulation model which is built using the Simscape

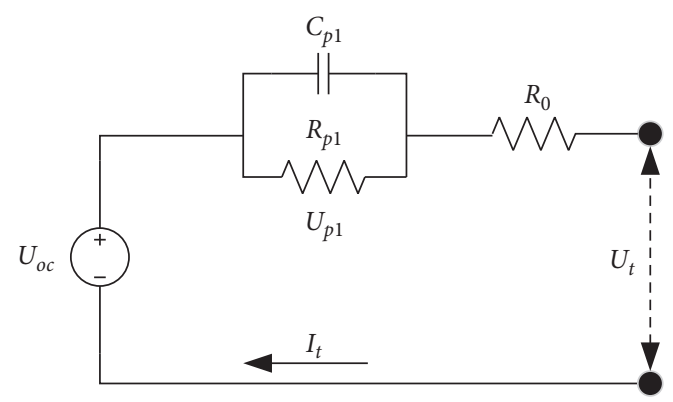

FIGURE 1: Schematic diagram of the first-order RC model.

Module Library. The current collected by the experimental equipment is input to the battery model, and the simulated voltage is obtained through simulation. Subsequently, the design optimization toolbox of Simulink is used to iteratively fit the simulated and actual voltage until the error accuracy between them meets the requirement $[35,36]$. This method can identify the battery model parameters corresponding to each SOC point at one time. Then, the corresponding relationship between SOC and model parameters is determined by lookup table.

Although this offline identification method can obtain accurate SOC value under specific conditions, these identified parameters are not necessarily suitable for any other working conditions. For example, when the model parameters identified under the Hybrid Pulse Power Characteristic (HPPC) condition are used in the Federal Urban Driving Schedule (FUDS) condition, the SOC error obtained by simulation is very large. This will be described in detail in this paper. Therefore, in order to solve this problem, this paper uses the FFRLS algorithm to identify the model parameters online.

Defining $E_{k}=U_{\mathrm{OC}}\left(\mathrm{SOC}_{k}\right)-U_{k}$, equation (4) can be transformed into the following form:

$$
\begin{aligned}
E_{k} & =\Lambda_{k-1} U_{p, k-1}+\left(1-\Lambda_{k-1}\right) R_{p} I_{k-1}+R_{0} I_{k} \\
& =\phi_{1} E_{k-1}+\phi_{2} I_{k}+\phi_{3} I_{k-1},
\end{aligned}
$$

where

$$
\left\{\begin{array}{l}
\phi_{1}=\Lambda_{k-1} \\
\phi_{2}=R_{0} \\
\phi_{3}=\left(1-\Lambda_{k-1}\right) R_{P}-\Lambda_{k-1} R_{0}
\end{array}\right.
$$

Assume the parameter identification system is a regression model, which can be described by the following equation:

$$
y_{k}=\varphi_{k} \xi_{k}
$$

where $y_{k}$ is the output of the system, $\varphi_{k}$ is the parameter matrix of the system, $\xi_{k}$ is the input matrix of the system:

$$
\left\{\begin{array}{l}
y_{k}=E_{k} \\
\varphi_{k}=\left[\begin{array}{lll}
\phi_{1} & \phi_{2} & \phi_{3}
\end{array}\right] \\
\xi_{k}=\left[\begin{array}{lll}
E_{k-1} & I_{k} & I_{k-1}
\end{array}\right]^{T}
\end{array}\right.
$$




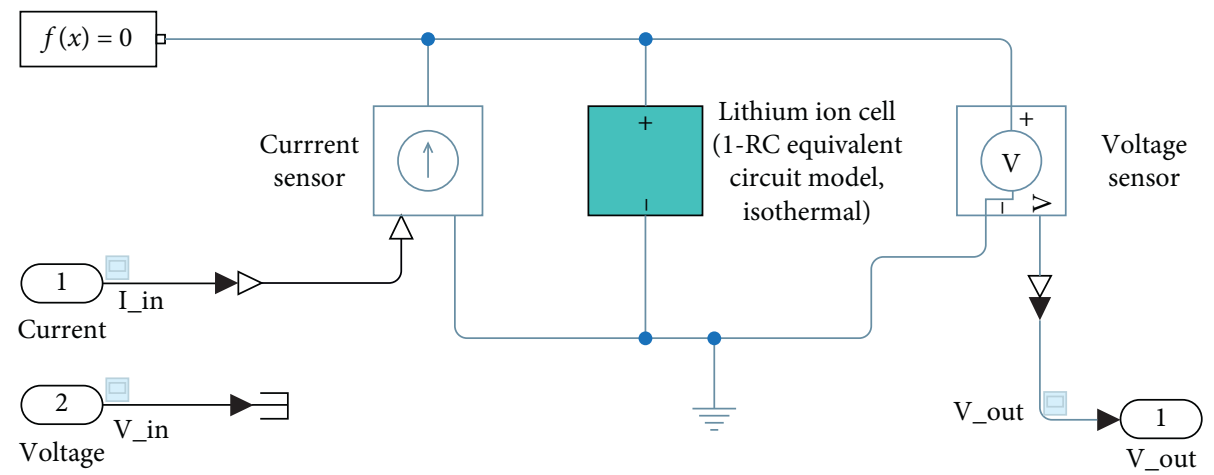

FIgURE 2: Simulation environment for parameter identification.

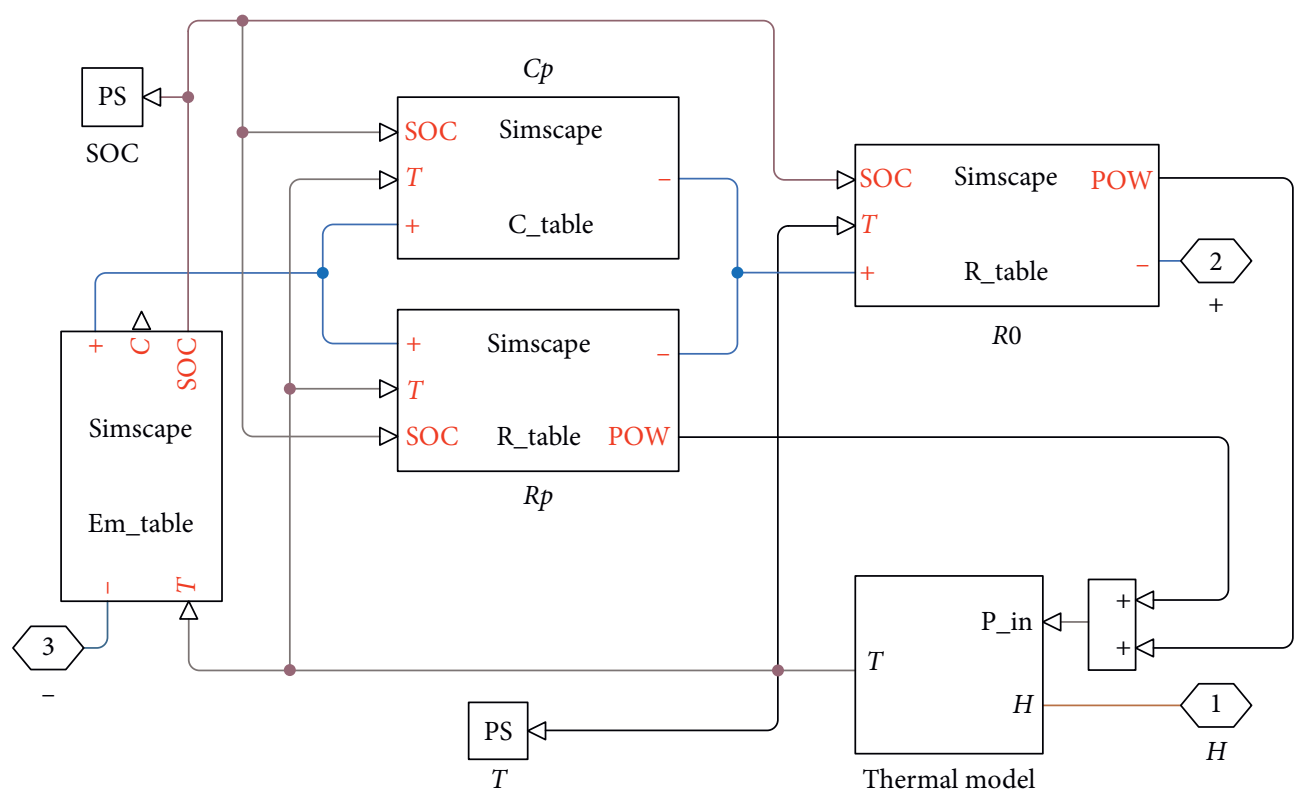

Figure 3: The Simscape module of the first-order RC battery model.

According to the actual current and voltage measured at times $k-1$ and $k$, the online parameters identification can be realized by the FFRLS method. The detailed identification process is as follows:

(i) Step 1. Initialization system parameter matrix $\varphi_{0}$, covariance matrix $P_{R, 0}$, forgetting factor $\lambda=0.98$.

(ii) Step 2. The gain matrix of FFRLS is calculated:

$$
K_{R, k}=\frac{P_{R, k-1} \xi_{k}}{\lambda+\xi_{k}^{T} P_{R, k-1} \xi_{k}} .
$$

(iii) Step 3. The covariance matrix of FFRLS is calculated:

$$
P_{R, k}=\lambda^{-1}\left(1-K_{R, k} \xi_{k}^{T}\right) P_{R, k-1}
$$

(iv) Step 4. The model prediction error is calculated:

$$
e_{R, k}=E_{k}-\xi_{k}^{T} \varphi_{k}
$$

(v) Step 5. The model parameter matrix is updated:

$$
\varphi_{k}=\varphi_{k-1}+K_{R, k} e_{R, k}
$$

(vi) Step 6. Separate model parameters from equations (7) and (9):

$$
\left\{\begin{array}{l}
R_{0, k}=\phi_{2, k}, \\
R_{P, k}=\frac{\phi_{1, k} \delta_{2, k}+\phi_{3, k}}{1-\phi_{1, k}}, \\
C_{P, k}=-\frac{\left(1-\phi_{1, k}\right) \Delta T}{\left(\phi_{1, k} \phi_{2, k}+\phi_{3, k}\right) \ln \phi_{1, k}} .
\end{array}\right.
$$




\section{Dual Extended Kalman Filter Method}

3.1. System Description. During actual charging and discharging, the battery SOC has fast time-varying characteristics, but the model parameters have slow time-varying characteristics. If the state and model parameters are updated at the same timescale, the calculation cost will be greatly increased. Moreover, frequent changes of parameters in the model will have a great impact on SOC prediction accuracy. Therefore, the dual-timescale method is used to update the parameters and SOC of the battery. The nonlinear system can be described by a mathematical formula as follows:

$$
\left\{\begin{array}{l}
\theta_{l}=\theta_{l-1}+w_{\theta, k-1}, \\
x_{k}=F\left(x_{k-1}, u_{k-1}, \theta_{l}\right)+w_{x, k-1}, \\
y_{k}=G\left(x_{k}, u_{k}, \theta_{l}\right)+v_{k},
\end{array}\right.
$$

where $x_{k}$ is the state of the system, $x=\left[\text { SOC } \cdot U_{P}\right]^{T} ; \theta$ is the model parameters of the battery system, $\theta_{l}=\left[R_{0} \cdot R_{P} \cdot C_{P}\right]^{T} ; k$ and $l$ describe the microtimescale and macrotimescale, respectively; $u$ is the input current value at $k ; y$ is the estimated voltage value in the system at $k ; w_{\theta, k-1}$ and $w_{x, k-1}$ are the Gaussian white noise of the parameters and state, respectively; and $v_{k}$ is the Gaussian white noise in the measurement equation.

3.2. EKF-AUKF Joint Estimation. EKF and UKF have achieved remarkable results in parameter identification and battery SOC estimation. In nonlinear system estimation, UKF does not need to conduct the first-order Taylor expansion of the equation to obtain the linearized model, but it uses the form of unscented transform to address the nonlinear transfer of mean and covariance. The performance of UKF is better than EKF in SOC estimation. However, the calculation amount of the EKF is less than that of the UKF [37]. In battery SOC estimation, the noise values $Q$ and $R$ of the standard UKF algorithm are considered fixed. However, in actual vehicle operation, the values are greatly affected by the external environment. The noise value is not constant, resulting in an increased effect on the estimated SOC value. To overcome this problem, this study uses covariance matching to update the process noise $Q$ and measurement noise $R$.

The parameters of the battery change slowly with time, and the SOC changes rapidly with time. Therefore, this study selects the AUKF to obtain accurate battery SOC on the microscale. The EKF is used to update the model parameters on the macroscale and decrease the calculation amount of the double Kalman filtering. During EKF algorithm updating of the parameters, redefining the timescale and setting a time constant value as $L_{\theta}, k=l \cdot L_{\theta}(l=1,2,3, \ldots)$ are necessary. If $k$ can be divided by $L_{\theta}$, then the parameters will not be updated from $k$ to $k+L_{\theta}$ and will be regarded as equal in this timescale. In SOC estimation using the AUKF algorithm, the covariance matrix of state and noise will be updated at every $k$ time. In order to better understand the working process of the two algorithms, the flow chart is shown in Figure 4. The detailed algorithm steps are as follows:

(i) Step 1. Initialization state $x$ and parameter $\theta$ and their corresponding covariance matrix and noise value $Q_{x, 0}$ $R_{x, 0} Q_{\theta, 0} \cdot R_{\theta, 0}$ :

$$
\begin{aligned}
\widehat{\theta}_{0}^{+} & =E\left(\theta_{0}\right), \\
P_{\theta, 0}^{+} & =E\left[\left(\theta_{0}-\widehat{\theta}_{0}^{+}\right)\left(\theta_{0}-\widehat{\theta}_{0}^{+}\right)^{T}\right], \\
\hat{x}_{0}^{+} & =E\left(x_{0}\right), \\
P_{x, 0}^{+} & =E\left[\left(x_{0}-\widehat{x}_{0}^{+}\right)\left(x_{0}-\widehat{x}_{0}^{+}\right)^{T}\right] .
\end{aligned}
$$

(ii) Step 2. Parameter estimation time update:

$$
\begin{aligned}
\widehat{\theta}_{l}^{-} & =\widehat{\theta}_{l-1}^{+}, \\
P_{\theta, l}^{-} & =P_{\theta, l-1}^{+}+Q_{\theta, l-1} .
\end{aligned}
$$

(iii) Step 3. State estimation time update.

(1) The weight of sampling points corresponding to UT transformation is calculated:

$$
\left\{\begin{array}{l}
\omega_{0}^{m}=\frac{\lambda}{(n+\lambda)}, \\
\omega_{0}^{c}=\frac{\lambda}{\left((n+\lambda)+\left(1-\alpha^{2}+\beta\right)\right)}, \\
\omega_{i}^{m}=\omega_{i}^{c}=\frac{1}{2(n+\lambda)}, \quad i=1 \sim 2 n, \\
\lambda=a^{2}(n+\kappa)-n .
\end{array}\right.
$$

(2) $2 n+1$ sigma point set is calculated:

$$
\begin{gathered}
\begin{cases}\chi_{k-1}^{0}=\hat{x}_{k-1}^{+}, & \\
\chi_{k-1}^{i}=\hat{x}_{k-1}^{+}+\sqrt{(n+\lambda) P_{x, k-1}^{+}}, & i=0,1,2, \ldots, n, \\
\chi_{k-1}^{i}=\hat{x}_{k-1}^{+}-\sqrt{(n+\lambda) P_{x, k-1}^{+}}, & i=n+1 \sim 2 n,\end{cases} \\
\chi_{k-1}^{i}=\left[\begin{array}{lll}
\hat{x}_{k-1}^{+} & \left.\hat{x}_{k-1}^{+}+\sqrt{(n+\lambda) P_{x, k-1}^{+}} \hat{x}_{k-1}^{+}-\sqrt{(n+\lambda) P_{x, k-1}^{+}}\right],
\end{array}\right.
\end{gathered}
$$

where $n$ is the state dimension of the battery, $\omega^{m}$ and $\omega^{c}$ are the corresponding weighting value of the mean and the covariance, respectively, and $\lambda$ is a scaling factor for reducing the total prediction error. The selection of $\alpha$ controls the distribution of the sampling point around the state. $\beta$ is a nonnegative parameter that can adjust the error of higher-order 


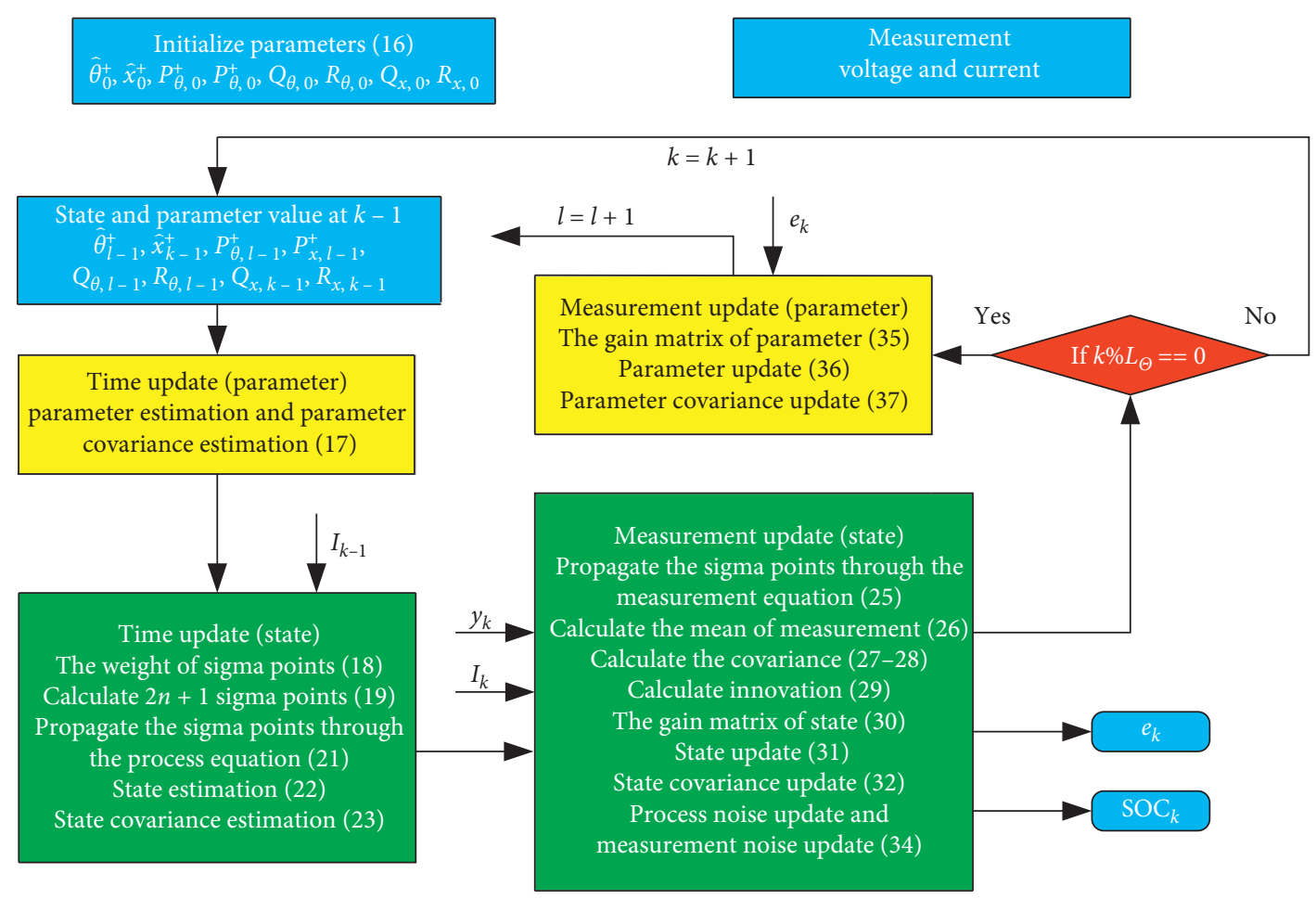

FIgURE 4: The flowchart of the SOC estimation and parameters update with EKF-AUKF algorithm.

terms, $\kappa$ is the secondary scaling parameter for ensuring that $(n+\lambda) P$ is a semipositive definite matrix. In this study, $n=3, \alpha=0.02, \beta=2$, and $\kappa=0$.

(3) One-step prediction of state values in the sigma point set:

$$
\chi_{k}^{i-}=F\left(\chi_{k-1}^{i}, \widehat{\theta}_{l}^{-}, u_{k-1}\right)
$$

(4) The mean and covariance are calculated on the basis of the one-step state prediction results:

$$
\begin{aligned}
\widehat{x}_{k}^{-} & =\sum_{i=0}^{2 n} \omega_{i}^{m} \chi_{k}^{i-}, \\
P_{x, k}^{-} & =\sum_{i=0}^{2 n} \omega_{i}^{c}\left(\chi_{k}^{i-}-\hat{x}_{k}^{-}\right)\left(\chi_{k}^{i-}-\hat{x}_{k}^{-}\right)^{T}+Q_{x, k-1} .
\end{aligned}
$$

(iv) Step 4. State measurement update.

(1) The state result of one-step prediction is transformed by UT again, and then a new sigma point set is generated:

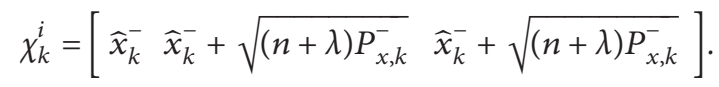

(2) The new set of state sigma points is substituted into the observation equation:

$$
\gamma_{k}^{i}=G\left(\chi_{k}^{i}, \widehat{\theta}_{l}^{-}, u_{k}\right)
$$

(3) The mean value and covariance of system prediction are calculated on the basis of the observation prediction value of the sigma point set:

$$
\begin{aligned}
\widehat{y}_{k}^{-} & =\sum_{i=0}^{2 n} \omega_{i}^{m} \gamma_{k}^{i}, \\
P_{x, k}^{y y} & =\sum_{i=0}^{2 n} \omega_{i}^{c}\left(\gamma_{k}^{i}-\hat{y}_{k}^{-}\right)\left(\gamma_{k}^{i}-\hat{y}_{k}^{-}\right)^{T}+R_{x, k}, \\
P_{x, k}^{x y} & =\sum_{i=0}^{2 n} \omega_{i}^{c}\left(\chi_{k}^{i-}-\widehat{x}_{k}^{-}\right)\left(\gamma_{k}^{i}-\widehat{y}_{k}^{-}\right)^{T} .
\end{aligned}
$$

(4) The innovation is calculated:

$$
e_{k}=y_{k}-\hat{y}_{k}^{-}
$$

(5) The Kalman gain matrix is calculated:

$$
K_{x, k}=P_{x, k}^{x y}\left(P_{x, k}^{y y}\right)^{-1} .
$$


(6) State and state covariance are updated:

$$
\begin{aligned}
\hat{x}_{k}^{+} & =\hat{x}_{k}^{-}+K_{x, k} e_{k}, \\
P_{x, k}^{+} & =P_{x, k}^{-}-K_{x, k} P_{x, k}^{y y} K_{x, k}^{T} .
\end{aligned}
$$

(7) Noise covariance is updated:

$$
\begin{aligned}
& F_{k}=\frac{1}{M} \sum_{i=k-M+1}^{k} e_{k} e_{k}^{T}, \\
& \left\{\begin{array}{l}
Q_{x, k}=K_{x, k} F_{k}\left(K_{x, k}\right)^{T}, \\
R_{x, k}=F_{k}-C_{x, k} P_{x, k}^{-}\left(C_{x, k}\right)^{T} .
\end{array}\right.
\end{aligned}
$$

(v) Step 5. Judging the timescale is necessary. If no remainder is found between $k$ and $L_{\theta}$, then the parameter measurement is updated in step (6). Otherwise, step (2) is repeated to continue the state update.

(vi) Step 6. Parameter measurement update: $k=l L_{\theta}$ $(l=1,2,3, \ldots)$ is conducted.

(1) The parameter gain matrix is calculated:

$$
K_{\theta, k}=P_{\theta, k}^{-} C_{\theta, k}^{T}\left(C_{\theta, k} P_{\theta, k}^{-} C_{\theta, k}^{T}+R_{\theta, k}\right)^{-1} .
$$

(2) Parameters and parameter covariance are updated:

$$
\begin{aligned}
\widehat{\theta}_{k}^{+} & =\widehat{\theta}_{k}^{-}+K_{\theta, k} e_{k}, \\
P_{\theta, k}^{+} & =\left(I-K_{\theta, k} C_{\theta, k}\right) P_{\theta, k}^{-} .
\end{aligned}
$$

It can be seen from the discrete equations (3) and (4) of the battery nonlinear system that the system input is current $I$, the output is voltage $U$, the state matrix is $x=$ [SOC $\left.U_{P}\right]^{T}$, and the parameter matrix is $\theta=\left[R_{0} \cdot R_{P} \cdot C_{P}\right]^{T}$. The correlation matrices used in the EKF-AUKF joint estimation algorithm are shown in equations (31)-(35). $C_{\theta, k}$ used in the parameter estimation can be derived from equations (36)-(41):

$$
\begin{aligned}
A_{k-1} & =\frac{F\left(\hat{x}_{k-1}^{+}, \hat{\theta}_{l}^{-}, u_{k-1}\right)}{\partial x}=\left[\begin{array}{cc}
1 & 0 \\
0 & \Lambda_{k-1}
\end{array}\right], \\
B_{k} & =\left[\begin{array}{c}
-\frac{\eta \Delta T}{C_{N}} \\
R_{p, l}\left(1-\Lambda_{k-1}\right)
\end{array}\right], \\
C_{x, k} & =\frac{G\left(\hat{x}_{k}^{-}, \hat{\theta}_{l}^{-}, u_{k}\right)}{\partial x}=\left[\frac{d U_{o c}\left(\mathrm{SOC}_{k}\right)}{d \mathrm{SOC}_{k}}-1\right],
\end{aligned}
$$

$$
\begin{aligned}
& D_{k}=\left[-R_{0}\right] \text {, } \\
& C_{\theta, k}=\left.\frac{\mathrm{d} G\left(\hat{x}_{k}^{-}, \widehat{\theta}, u_{k}\right)}{\mathrm{d} \theta}\right|_{x=\widehat{x}_{k}^{-}}, \\
& \frac{\mathrm{d} G\left(\hat{x}_{k}^{-}, \widehat{\theta}, u_{k}\right)}{\mathrm{d} \theta}=\frac{\partial G\left(\hat{x}_{k}^{-}, \hat{\theta}, u_{k}\right)}{\partial \theta} \\
& +\frac{\partial G\left(\widehat{x}_{k}^{-}, \widehat{\theta}, u_{k}\right)}{\partial \widehat{x}_{k}^{-}} \frac{\mathrm{d} \widehat{x}_{k}^{-}}{\mathrm{d} \theta} \\
& \frac{\mathrm{d} \widehat{x}_{k}^{-}}{\mathrm{d} \theta}=\frac{\partial F\left(\hat{x}_{k-1}^{+}, \widehat{\theta}, u_{k-1}\right)}{\partial \theta} \\
& +\frac{\partial F\left(\hat{x}_{k-1}^{+}, \widehat{\theta}, u_{k-1}\right)}{\partial \widehat{x}_{k-1}^{+}} \frac{\mathrm{d} \widehat{x}_{k-1}^{+}}{\mathrm{d} \theta} \\
& \frac{\mathrm{d} \widehat{x}_{k-1}^{+}}{\mathrm{d} \theta}=\frac{\mathrm{d} \widehat{x}_{k-1}^{-}}{\mathrm{d} \theta}-K_{x, k-1} \frac{\mathrm{d} G\left(\hat{x}_{k-1}^{-}, \widehat{\theta}, u_{k-1}\right)}{\mathrm{d} \theta}, \\
& \frac{\partial G\left(\hat{x}_{k}^{-}, \widehat{\theta}, u_{k}\right)}{\partial \theta}=\left[\begin{array}{lll}
-I_{k} & 0 & 0
\end{array}\right],
\end{aligned}
$$

$$
\frac{\partial F\left(\hat{x}_{k-1}^{+}, \hat{\theta}, u_{k-1}\right)}{\partial \theta}=\left[\begin{array}{ccc}
0 & 0 & 0 \\
0 & a_{1} & a_{2}
\end{array}\right],
$$

$$
\left\{\begin{array}{l}
a_{1}=\frac{\Delta T}{R_{p, l}^{2} C_{p, l}} \Lambda U_{p, k-1}+[1-\Lambda] I_{k-1}-\frac{\Delta T}{R_{p, l} C_{p, l}} \Lambda I_{k-1} \\
a_{2}=\frac{\Delta T}{R_{p, l} C_{p 1, l}^{2}} \Lambda U_{p, k-1}-\frac{\Delta T}{C_{p, l}^{2}} \Lambda I_{k-1} .
\end{array}\right.
$$

\section{Experiment and Parameter Identification}

4.1. Experimental Data Description. This study uses the open-source dataset provided by the University of Maryland. Figure 5 shows the experimental equipment. The detailed experimental process is described in [38]. The experimental equipment is mainly composed of a charge and discharge test system for batteries (Arbin BT2000), a thermal chamber for temperature control, and a host computer. INR 18650-20R lithium-ion battery is used as the experimental object. Its rated capacity is $2000 \mathrm{mAh}$, rated voltage is $3.6 \mathrm{~V}$, charging cut-off voltage is $4.2 \mathrm{~V}$, and discharging cut-off voltage is $2.5 \mathrm{~V}$. Through the test software installed on the computer for controlling the Arbin BT2000 system and thermal chamber, the battery charge and discharge tests are conducted at different temperatures and sophisticated dynamic current profiles, and the experimental data are collected. All charging and discharging conditions were tested at $0^{\circ} \mathrm{C}, 25^{\circ} \mathrm{C}$, and $45^{\circ} \mathrm{C}$, and the sampling time was $1 \mathrm{~s}$. 


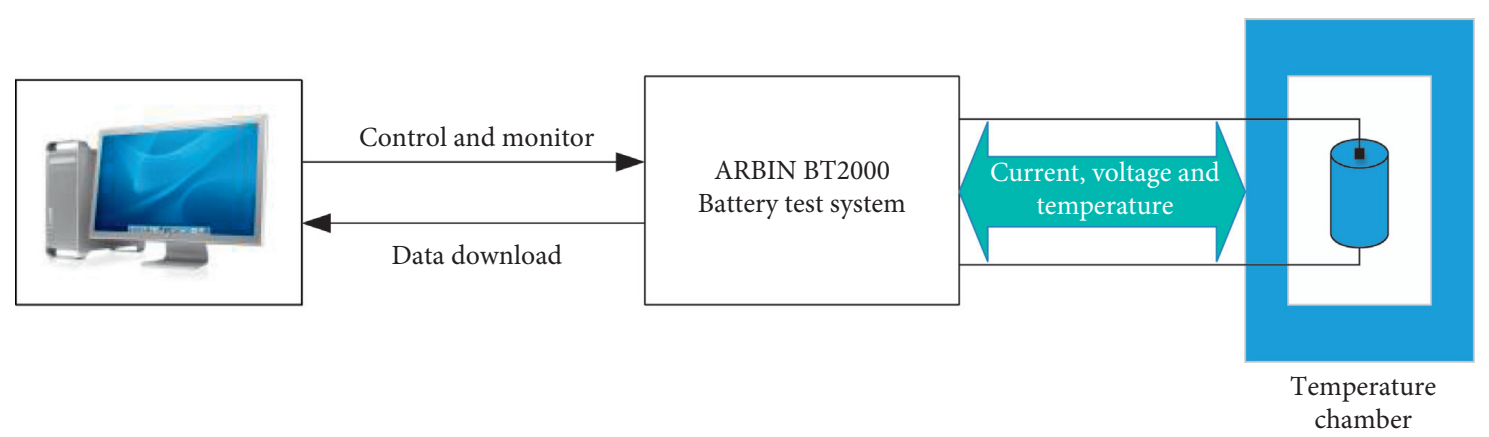

FIGURE 5: Schematic of the battery experiment bench.

4.2. Incremental OCV Test. At present, three classic ways are used to obtain the SOC-OCV relationship. Incremental [39] and low-current [40] OCV test methods are offline parameter identification methods for obtaining the SOCOCV relationship. The two methods are compared in detail in [38], and the experimental results indicate that the incremental test method is more stable than the low-current test method. In addition, the SOC-OCV relationship is regarded as a part of the model parameters. The FFRLS or Kalman filter algorithm is used for online parameter identification. This method is adopted for online SOC estimation and parameter update [22]. The SOC-OCV relationship obtained by this method cannot be affected by the temperature and charging and discharging conditions. However, too many parameters will bring inconvenience to algorithm debugging, and the uncertainty of the initial value of parameters will affect the SOC accuracy. Therefore, this study selects the incremental OCV test method to conduct the following experiments.

Taking room temperature $\left(25^{\circ} \mathrm{C}\right)$ as an example, the battery was discharged under the HPPC condition. (1) The battery was fully charged using the standard constant current and constant voltage (CC-CV) charging mode, and then it was at rest for $120 \mathrm{~min}$. At this time, the SOC value was regarded as 1 , and the measured battery terminal voltage value was recorded as OCV value. (2) The battery was discharged at a constant current of $1 \mathrm{~A}$, the current was unloaded when discharging to $10 \%$ of the rated capacity, and then the battery was at rest for $120 \mathrm{~min}$ to eliminate the polarization effect. At this time, the measured battery terminal voltage was recorded as the $\mathrm{OCV}$ at $\mathrm{SOC}=0.9$. (3) Step (2) is repeated and the OCV corresponding to each SOC point in turn is recorded. (4) When the battery was discharged to the cut-off voltage. Figure 6(a) presents the current and voltage during the entire experiment. The fitting curves of SOC and OCV at three temperatures are shown in Figure 6(b), and the fitting coefficients are shown in Table 1.

4.3. Parameter Identification. According to two kinds of model parameter identification methods, the first method uses the ordinary RLS method to identify the parameters of the battery through the HPPC working condition data, and the second method uses the FFRLS method to identify the parameters of the battery through the FUDS working condition data. Figure 7 shows the current and voltage profiles of the FUDS condition.

The results of the model parameters identified by the two methods are shown in Figures 8-10. The figure presents that the $R_{0}$ identified by the two operating conditions at three different temperatures is basically stable. At the same temperature, the identification results of $R_{0}$ show a huge difference between the two conditions, and the identification results of FUDS condition are obviously smaller than those of HPPC condition. From the results of parameter identification under three different temperatures, the change range of $R_{P}$ is larger than that of $R_{0}$. The identification results of $C_{P}$ under the FUDS condition are more stable than those under HPPC condition. Through the above analysis and discussion, it indicates that the parameter identification results of FUDS working condition are relatively stable. Then, the average value of the identification results of FUDS is taken as the initial model parameter of the proposed algorithm, as shown in Table 2.

As illustrated in Figure 11(a), the simulated voltages at three temperatures well track the actual voltages. The voltage errors in Figure 11(b) indicate that the mean absolute error of the voltage is within $0.005 \mathrm{~V}$ when the temperature is $25^{\circ} \mathrm{C}$ and $45^{\circ} \mathrm{C}$, and the mean absolute error of voltage is within $0.008 \mathrm{~V}$ when the temperature is $0^{\circ} \mathrm{C}$. The absolute voltage error is within $0.04 \mathrm{~V}$ in most discharge cycles at three temperatures, and the voltage error is relatively large only in the last discharge cycle. Therefore, the parameter identification results of HPPC can meet the simulation requirements of this working condition. Whether the parameter identification results of FUDS can meet the simulation requirements of the working condition will be discussed in the next section.

\section{Experiment Verification and Discussion}

The SOC prediction performance of this method is verified through the FUDS test dataset. During actual work, less than $10 \%$ of the battery capacity is rarely used. If the battery is continuously used, then the battery life is shortened. Therefore, all dynamic data used in this study are $80 \%-10 \%$ of the rated capacity. The actual SOC value in this study is obtained by the ampere-hour integral method, and its initial actual value is 0.8 . 


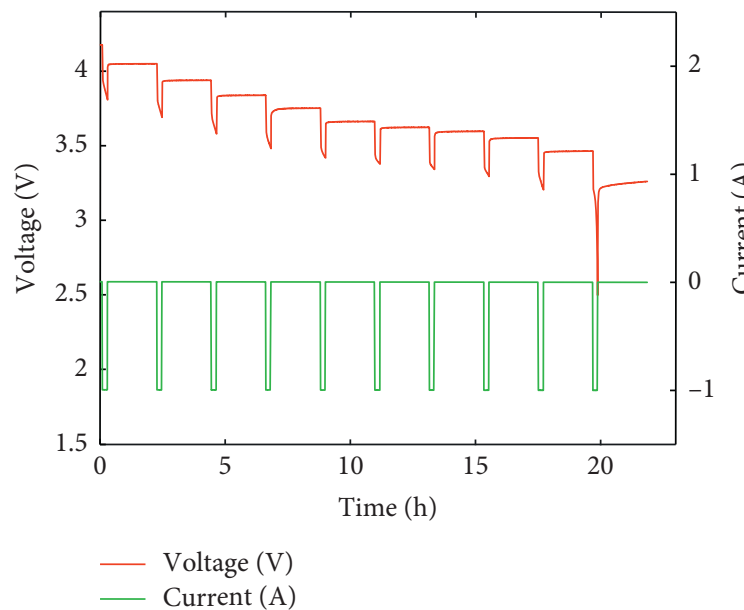

(a)

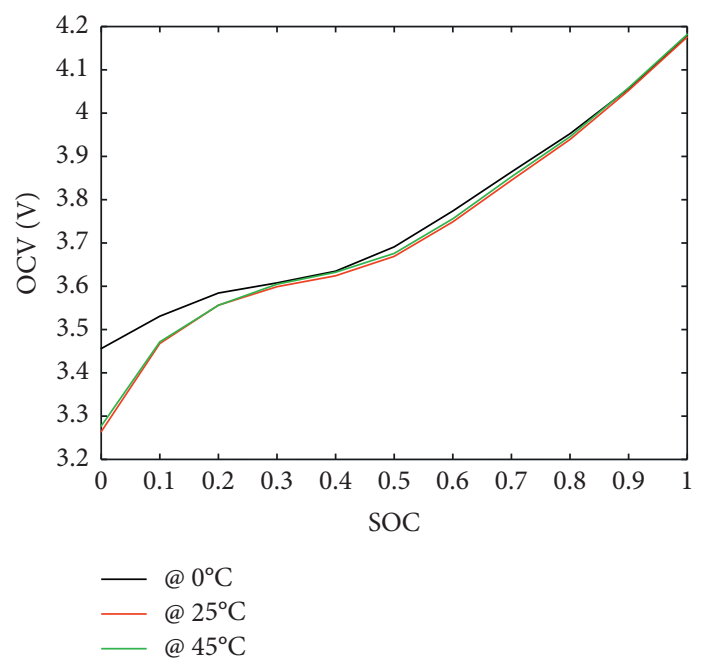

(b)

FIGURE 6: Experimental results of incremental OCV test: (a) the voltage and current profiles; (b) the OCV-SOC curves of three temperatures.

TABLE 1: The fitting coefficients of OCV-SOC.

\begin{tabular}{lccccccccc}
\hline$T\left({ }^{\circ} \mathrm{C}\right)$ & $H_{1}$ & $H_{2}$ & $H_{3}$ & $H_{4}$ & $H_{5}$ & $H_{6}$ & $H_{7}$ & $H_{8}$ \\
\hline 0 & -36.68 & 103.712 & -77.889 & -31.913 & 70.152 & -31.331 & 4.083 & 0.587 & 3.456 \\
25 & -173.48 & 680.209 & -1077.8 & 887.818 & 412.88 & 115.548 & -21.91 & 3.408 & 3.264 \\
45 & -232.09 & 923.694 & -1491.6 & 1256.828 & 596.086 & 164.555 & -27.931 & 3.578 & 3.277 \\
\hline
\end{tabular}

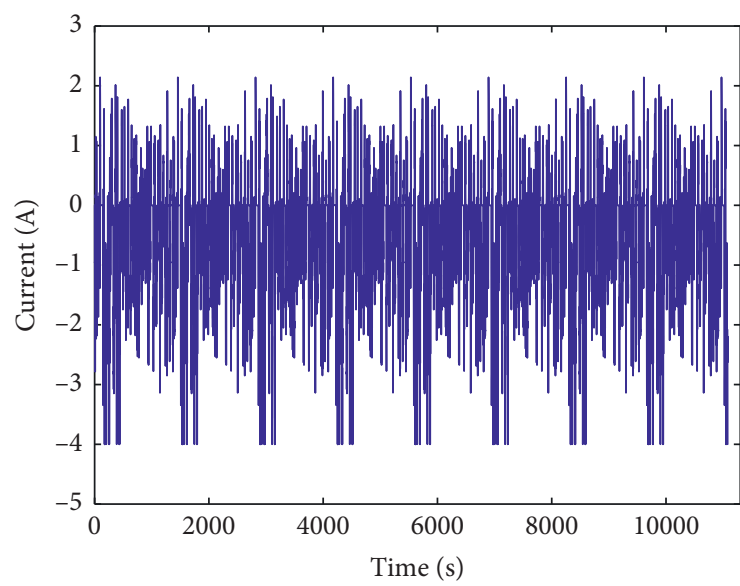

(a)

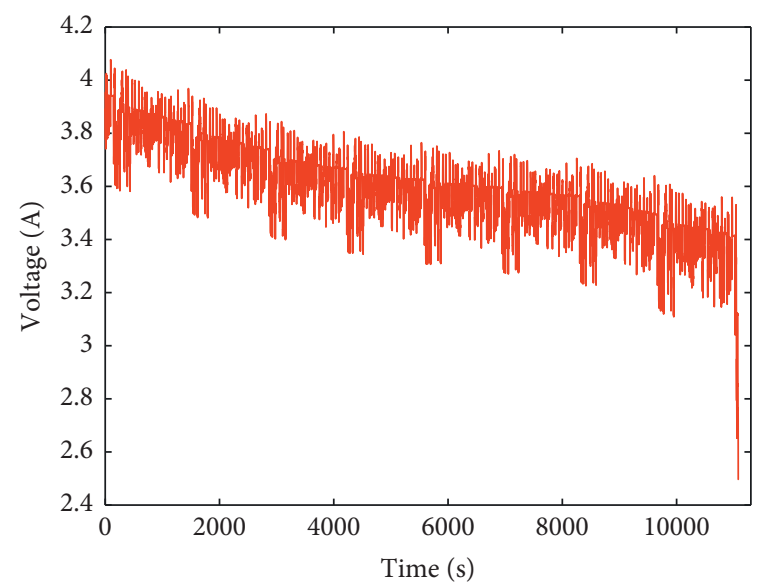

(b)

FIGURE 7: FUDS dynamic condition test: (a) current profiles and (b) voltage profiles.

5.1. Estimation Results with Determined Initial SOC Value. To verify whether the parameters identified by the twoparameter identification methods can be applied to the FUDS condition, the EKF algorithm is applied to predict the battery SOC and load voltage. According to the parameters identified by the HPPC condition, a battery model is built for simulation by looking up the table; the model is recorded as EKF-1. According to the average parameter identified by the FUDS condition, a battery model is built for simulation with fixed parameters; the model is recorded as EKF-2. At the same time, the parameter values in Table 2 are taken as the initial values of the battery. Then, online parameter identification and SOC estimation can be realized by EKF-UKF and EKFAUKF algorithms. To avoid the influence of temperature on the estimated results of each algorithm, the parameters used in these algorithms are the model parameters identified under their own temperature. The initial SOC values of the four algorithms are the same as the reference SOC initial values, that is, SOC is 0.8 . In order to compare the SOC and voltage prediction performance of the four algorithms in the entire FUDS test, the mean absolute 


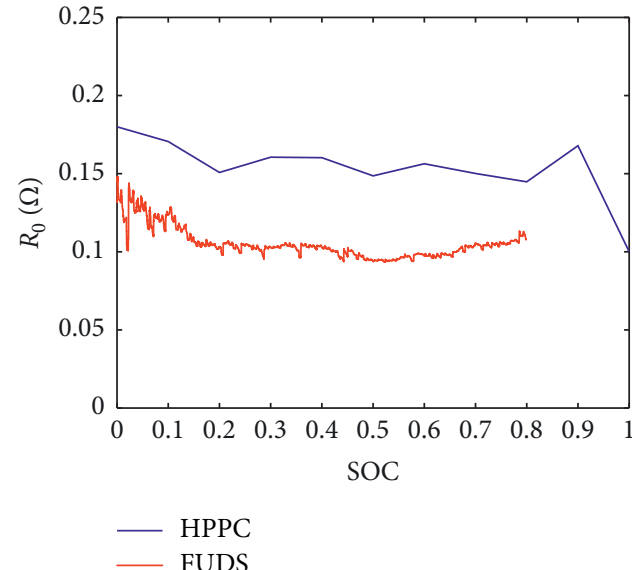

(a)

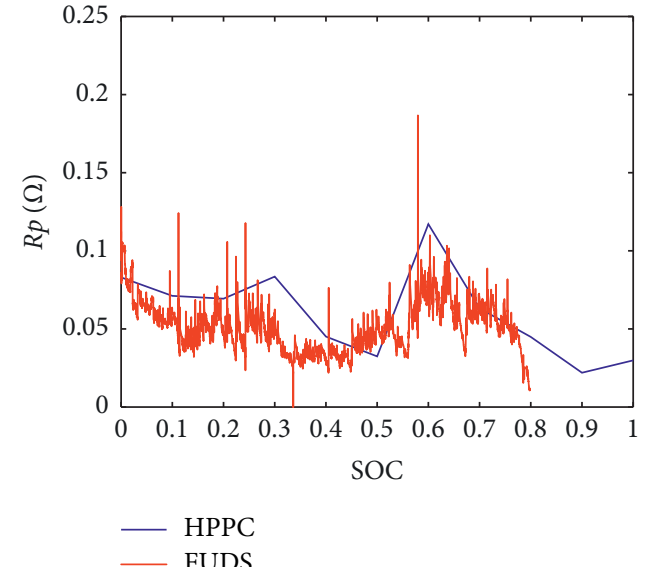

(b)

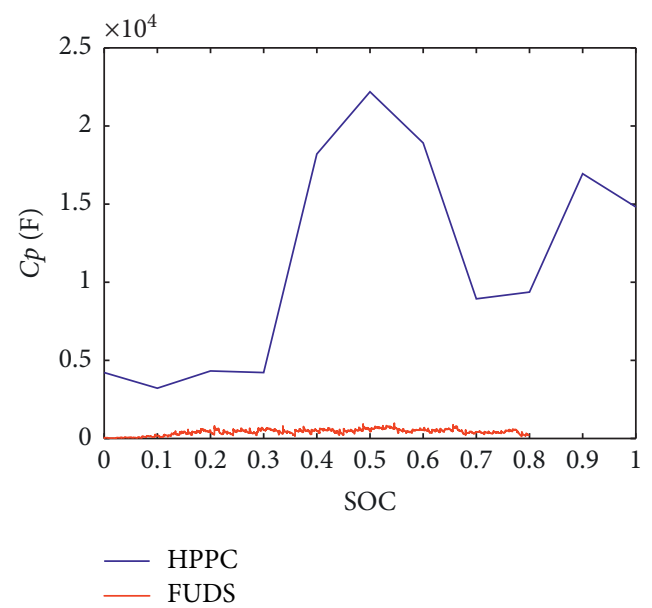

(c)

Figure 8: Parameter identification results under two working conditions at $0^{\circ} \mathrm{C}$ : (a) R0; (b) RP; and (c) CP.

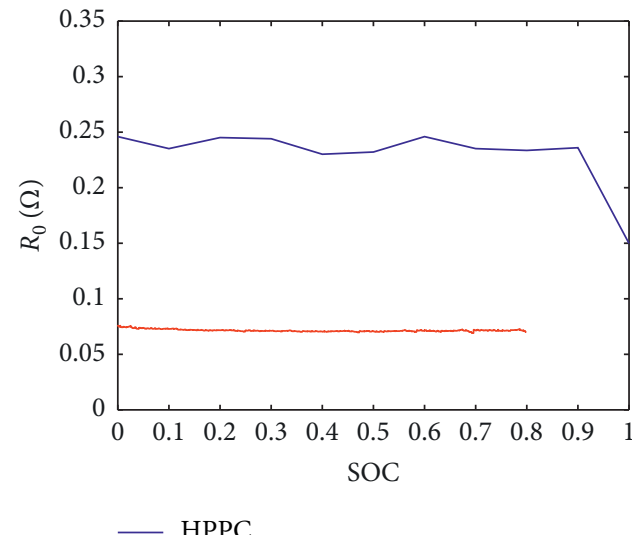

PPC

FUDS

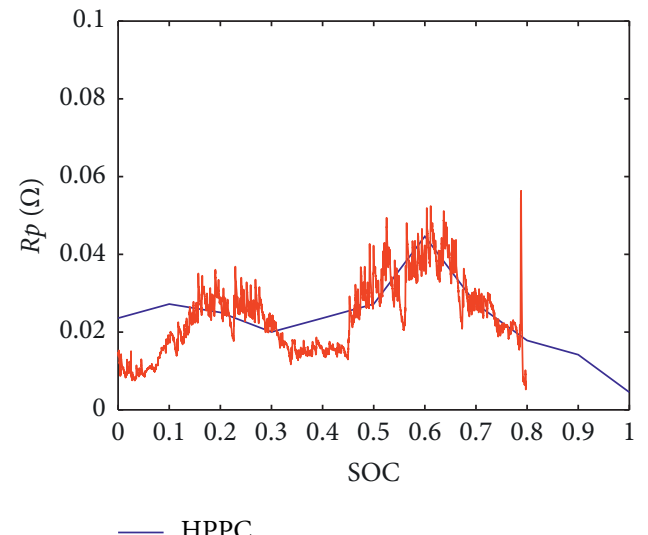

- FUDS

(a)

(b)

FIgURE 9: Continued. 


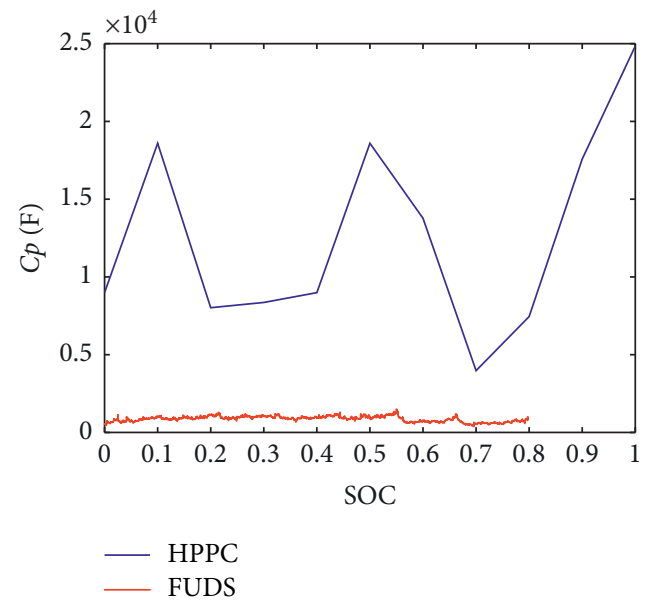

(c)

FIgURE 9: Parameter identification results under two working conditions at $25^{\circ} \mathrm{C}$ : (a) R0; (b) RP; and (c) CP.

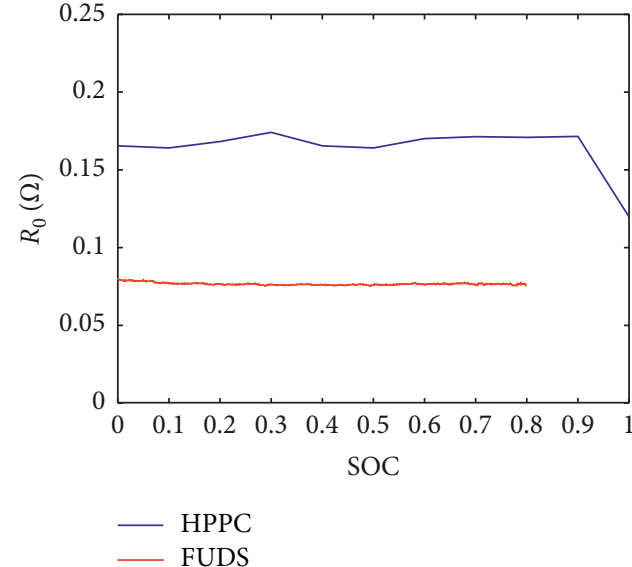

(a)

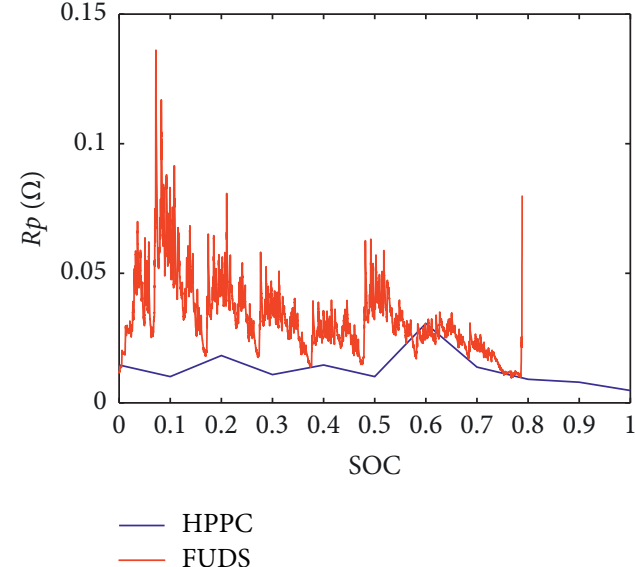

(b)

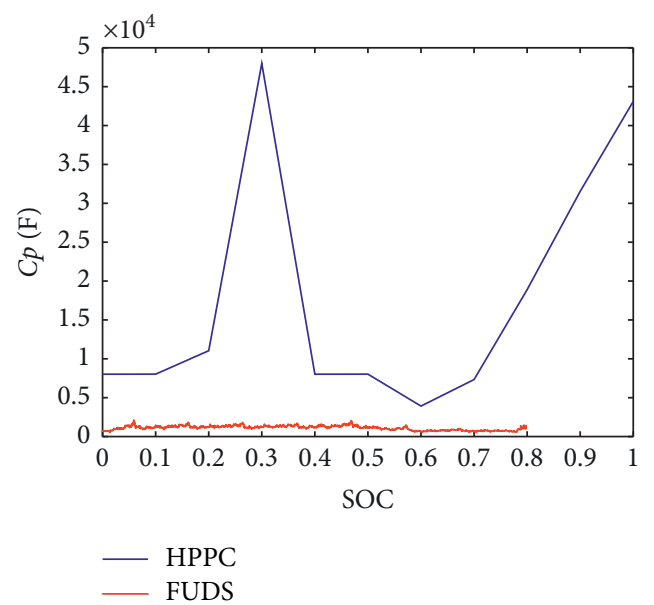

(c)

Figure 10: Parameter identification results under two working conditions at $45^{\circ} \mathrm{C}$ : (a) R0; (b) RP; and (c) CP. 
TABLE 2: Model parameters identification under FUDS condition.

\begin{tabular}{lllr}
\hline$T\left({ }^{\circ} \mathrm{C}\right)$ & $R_{0}(\Omega)$ & $R_{P}(\Omega)$ & $C_{P}(\mathrm{~F})$ \\
\hline 0 & 0.1004 & 0.0493 & 492.0793 \\
25 & 0.0713 & 0.0264 & 887.5052 \\
45 & 0.0763 & 0.0291 & 1110.036 \\
\hline
\end{tabular}
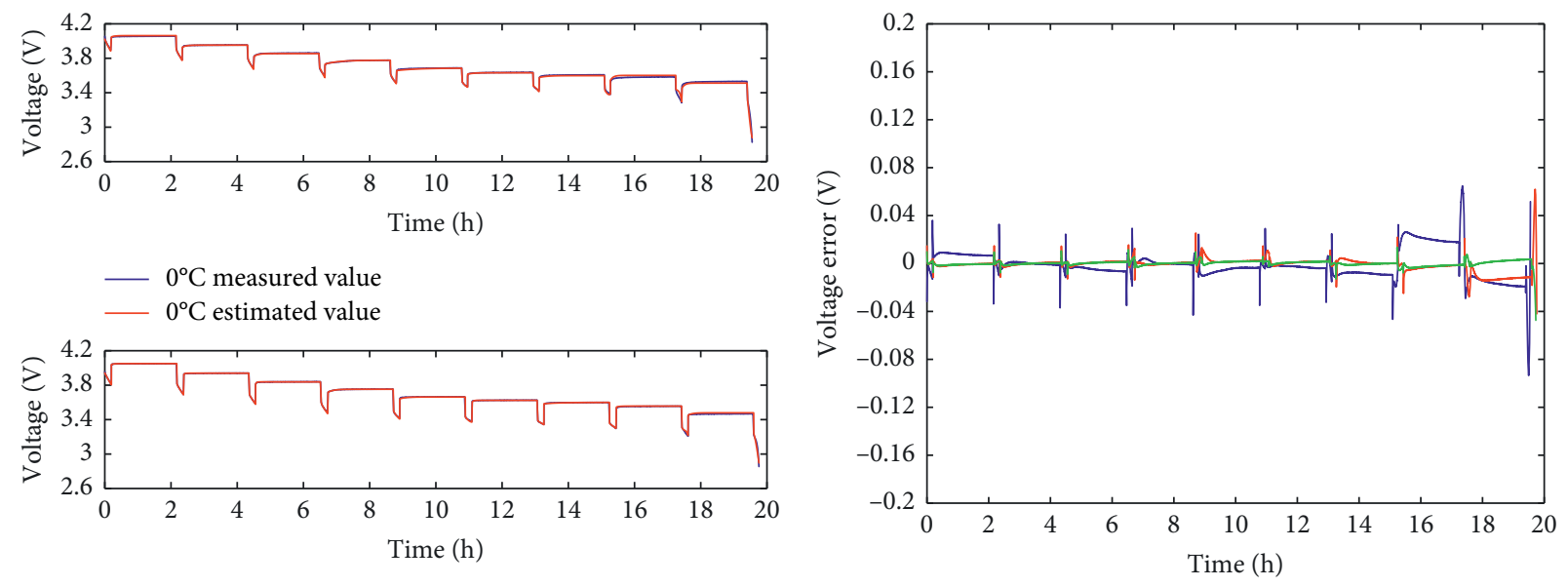

- $25^{\circ} \mathrm{C}$ measured value

$25^{\circ} \mathrm{C}$ estimated value

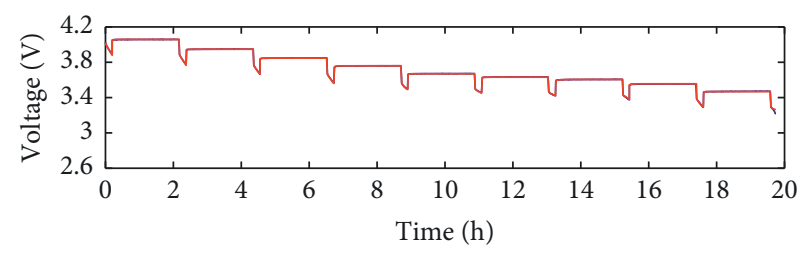

$-@ 0^{\circ} \mathrm{C}$

- @ $25^{\circ} \mathrm{C}$

- @ $45^{\circ} \mathrm{C}$

- $45^{\circ} \mathrm{C}$ measured value

- $45^{\circ} \mathrm{C}$ estimated value

(a)

(b)

FIgURE 11: Simulation results under HPPC condition: (a) voltage simulation results and (b) voltage simulation error.

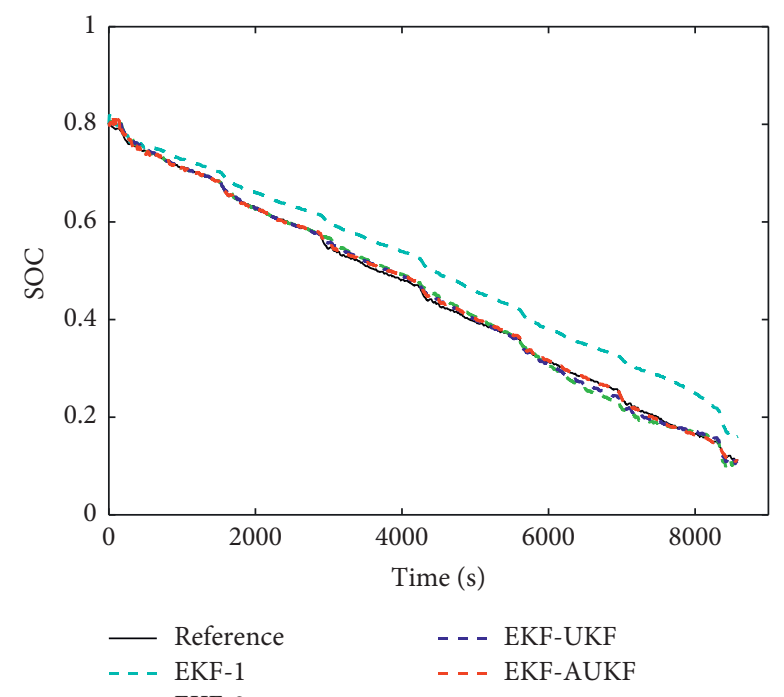

(a)

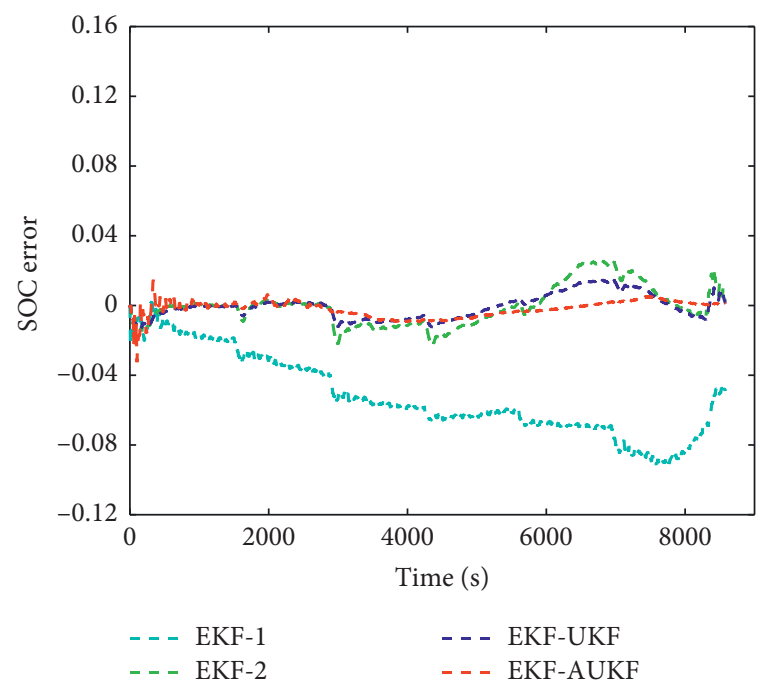

(b)

Figure 12: Continued. 


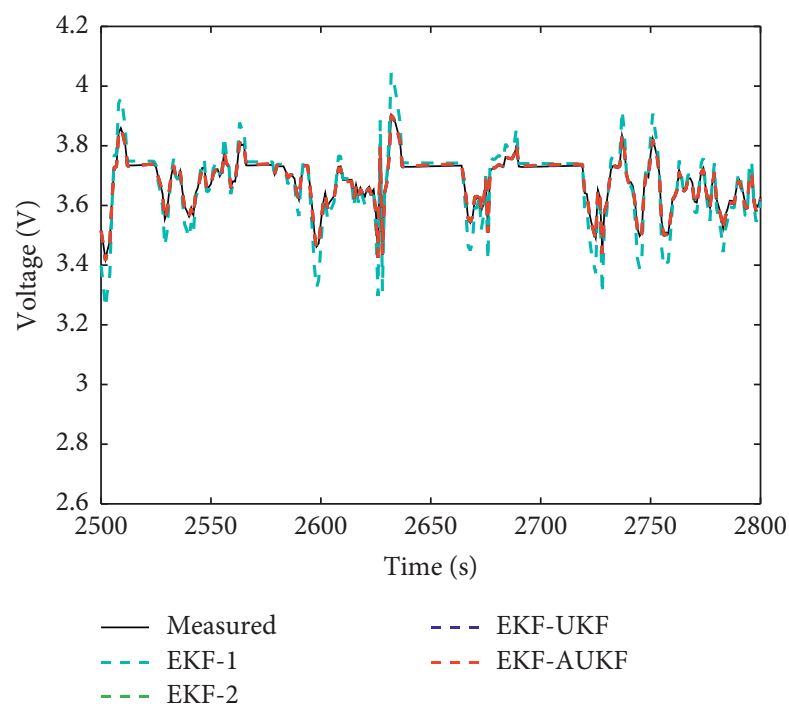

(c)

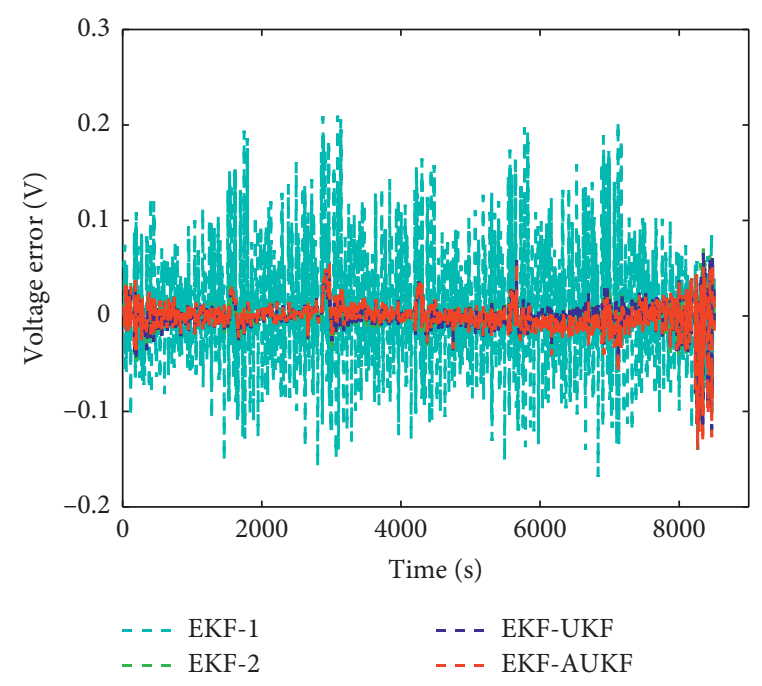

(d)

FIGURE 12: The comparison of estimation results at $0^{\circ} \mathrm{C}$ : (a) prediction results of SOC; (b) SOC error; (c) prediction results of voltage; and (d) voltage error.

error (MAE) and root mean square error (RMSE) are introduced as indicators for comparison.

Figures 12(a)-12(d) show the SOC and voltage estimated by the four algorithms at $0^{\circ} \mathrm{C}$. Specifically, Figure $12(\mathrm{a})$ presents that the SOC value estimated by the EKF-1 algorithm deviates from the true SOC value, whereas the results obtained by the other three algorithms are consistent with the reference SOC curve. Figure 12(c) illustrates that the voltage value estimated by the EKF-1 algorithm deviates from the measured value, whereas the voltage values estimated by the three other algorithms are consistent with the measured value. In the entire FUDS test, the SOC RMSEs estimated by the four algorithms are 5.7\%, 1.117\%, 0.705\%, and $0.496 \%$, respectively. The voltage RMSEs estimated by the four algorithms are 46.135, 13.129, 13.217, and $13.755 \mathrm{mV}$, respectively. Figures $13(\mathrm{a})-13(\mathrm{~d})$ show the SOC and voltage values estimated by the four algorithms at $25^{\circ} \mathrm{C}$. Figures 13(b) and 13(d) illustrate that the SOC and voltage errors estimated by EKF-1 are the largest, whereas the three other estimation methods have good SOC and voltage estimation results. In the entire FUDS test, the SOC RMSEs estimated by the four algorithms are $14.078 \%, 0.675 \%$, $0.528 \%$, and $0.437 \%$, respectively. The voltage RMSEs estimated by the four algorithms are 161.18, 4.791, 5.061, and $4.23 \mathrm{mV}$, respectively. Figures $14(\mathrm{a})-14(\mathrm{~d})$ show the SOC and voltage values estimated by the four algorithms at $45^{\circ} \mathrm{C}$. In the entire FUDS test, the SOC RMSEs estimated by the four algorithms are $7.391 \%, 0.767 \%, 0.514 \%$, and $0.461 \%$, respectively. The voltage RMSEs estimated by the four algorithms are 90.608, 5.501, 4.801, and $4.505 \mathrm{mV}$, respectively.

Comparing the simulation results of EKF-1 and EKF-2 suggests that although the model parameters identified through the HPPC operating condition have achieved accurate voltage simulation results under HPPC condition, the simulation results under the FUDS condition perform poorly. Therefore, the battery model parameters identified by the HPPC condition are inapplicable to any condition. Due to the limited dataset, this study does not discuss in detail the effect of the chargedischarge ratio on parameter identification under the HPPC condition. However, the three other estimation methods have achieved good results at three temperatures. The model parameters identified by FFRLS can be applied to dynamic conditions. The statistical results in Table 3 show that the SOC estimation accuracy of the EKF-UKF and EKF-AUKF algorithms is higher than that of the EKF algorithm at different temperatures. The real-time parameters updated online by the double Kalman filter algorithm are more accurate than the SOC values estimated by the fixed parameters. The accuracy of SOC estimation obtained by the EKF-AUKF is higher than that of the EKF-UKF. The proposed algorithm can improve the prediction performance of SOC by updating the noise value and the covariance matrix.

5.2. Experiment Results with Initial SOC Errors. Obtaining an accurate initial SOC value during actual work is difficult for a vehicle. Therefore, it is important to verify the robustness of the proposed algorithm in the case of an unknown initial SOC value. The initial SOC values were set to 0.9 , $0.7,0.5$, and 0.3 , respectively. The dataset used in this part is the experimental data of FUDS at $25^{\circ} \mathrm{C}$. Figures 15 (a) and 15(b) show the SOC estimation and error results for different initial SOC values. Figures 15(c) and 15(d) present the voltage estimation and error results for different initial SOC values. As can be seen from Figure 15, the predicted SOC and voltage curves converge quickly to the true value within 50-80 s under different initial SOC errors. The larger the initial SOC error, the slower the convergence speed. The RSME of the SOC is within $1 \%$, whereas that of the voltage is within $0.01 \mathrm{~V}$ at each SOC 


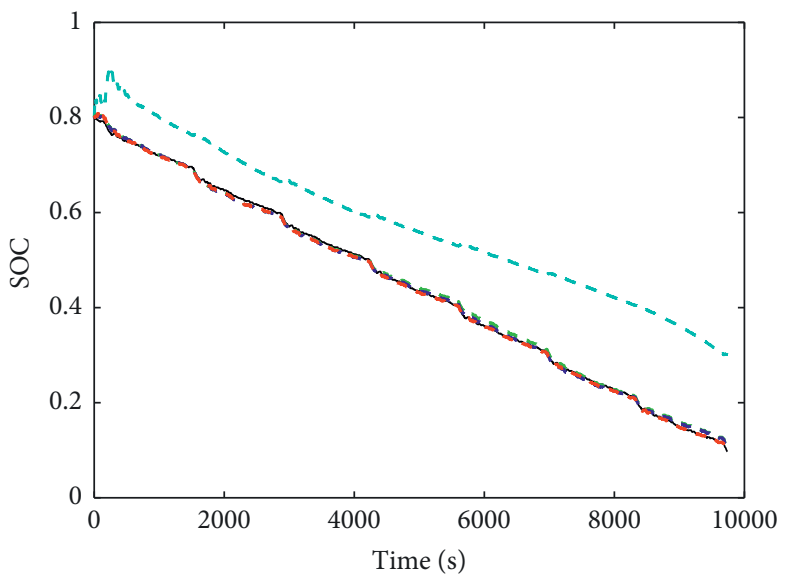

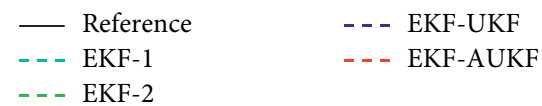

(a)

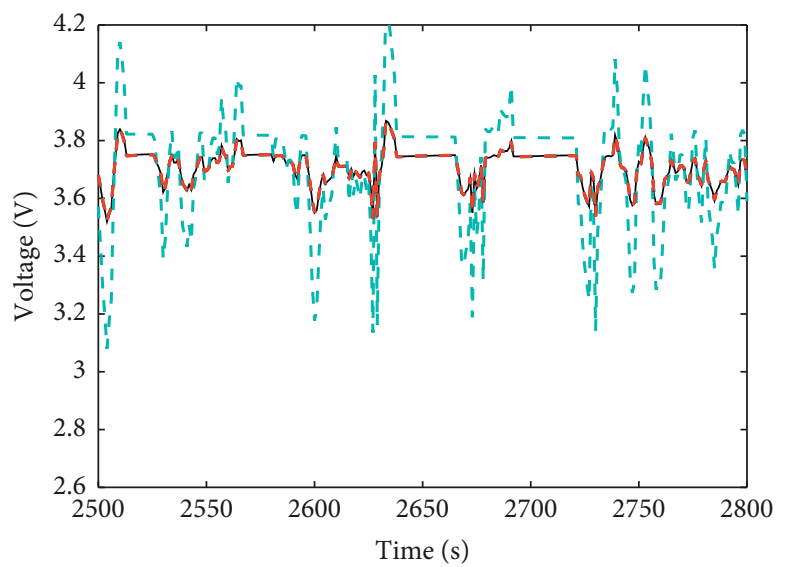

- Measured
--- EKF-1
--- EKF-2

- - - EKF-UKF

- . EKF-AUKF

(c)

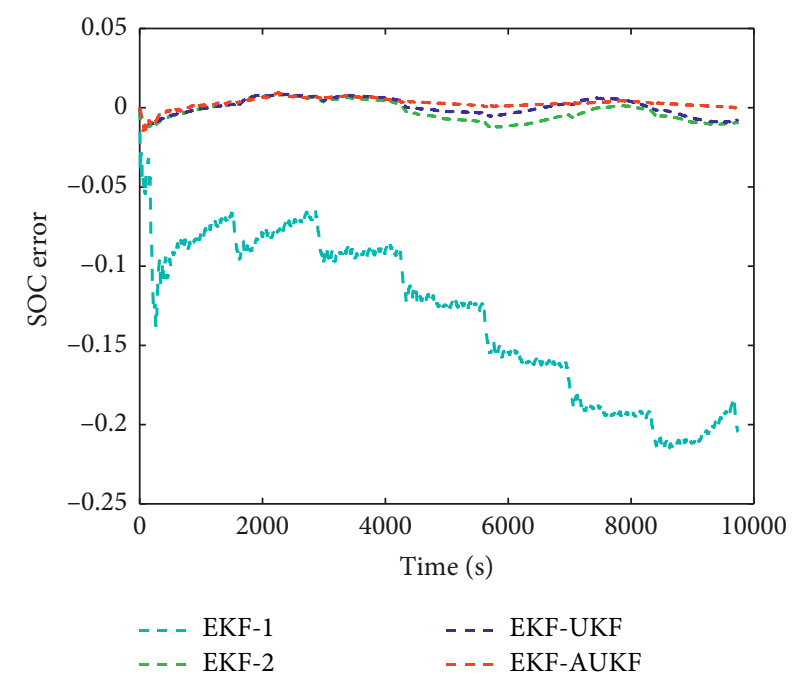

(b)

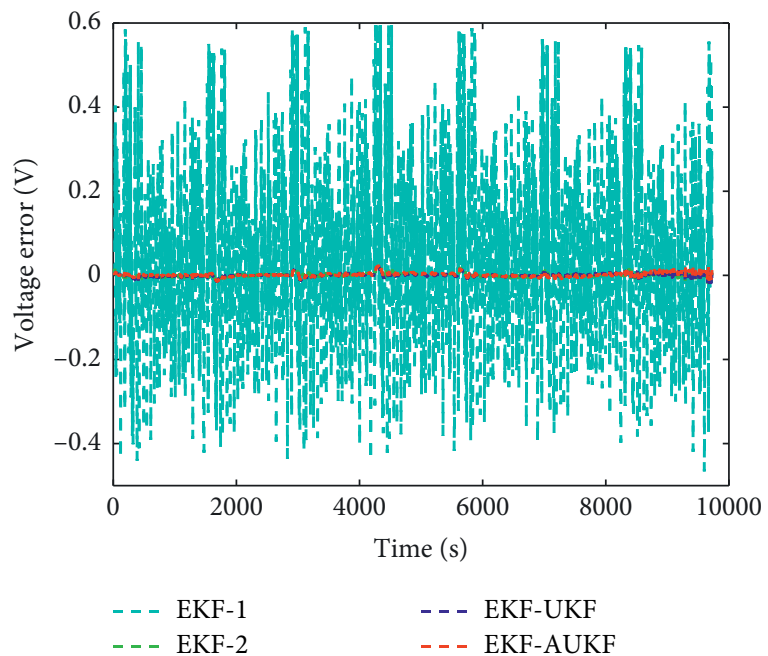

(d)

FIGURE 13: The comparison of estimation results at $25^{\circ} \mathrm{C}$ : (a) prediction results of SOC; (b) SOC error; (c) prediction results of voltage; and (d) voltage error.

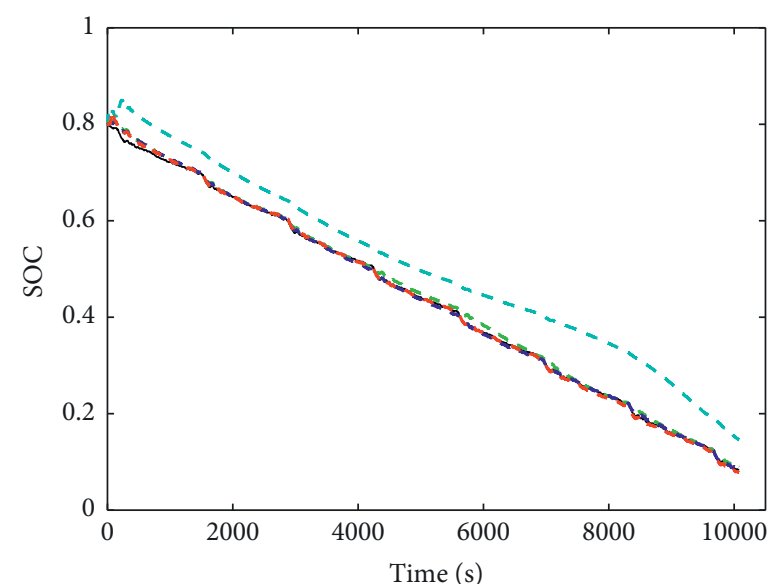

$\begin{array}{ll}\text { - Reference } & -- \text { EKF-UKF } \\ - \text { - EKF-1 } & --- \text { EKF-AUKF } \\ - \text { - EKF-2 } & \end{array}$

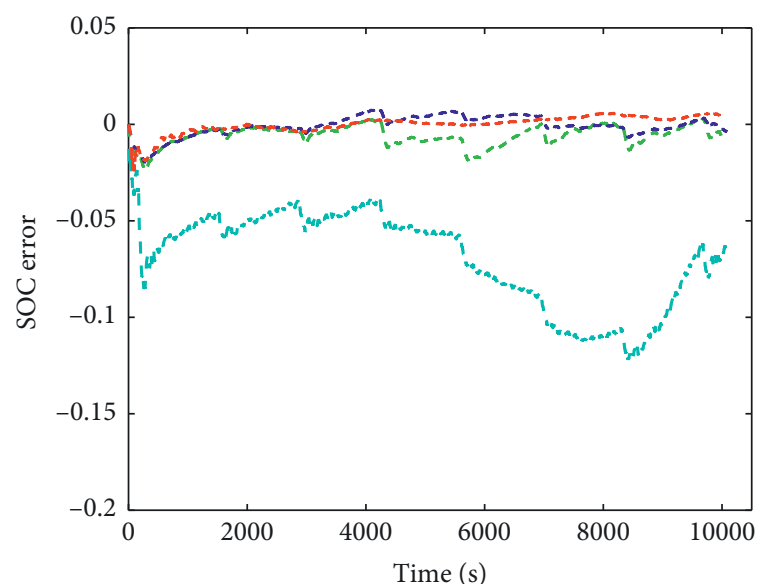

$$
\begin{array}{ll}
--- \text { EKF-1 } & -- \text { EKF-UKF } \\
--- \text { EKF-2 } & --- \text { EKF-AUKF }
\end{array}
$$

(a)

(b)

Figure 14: Continued. 


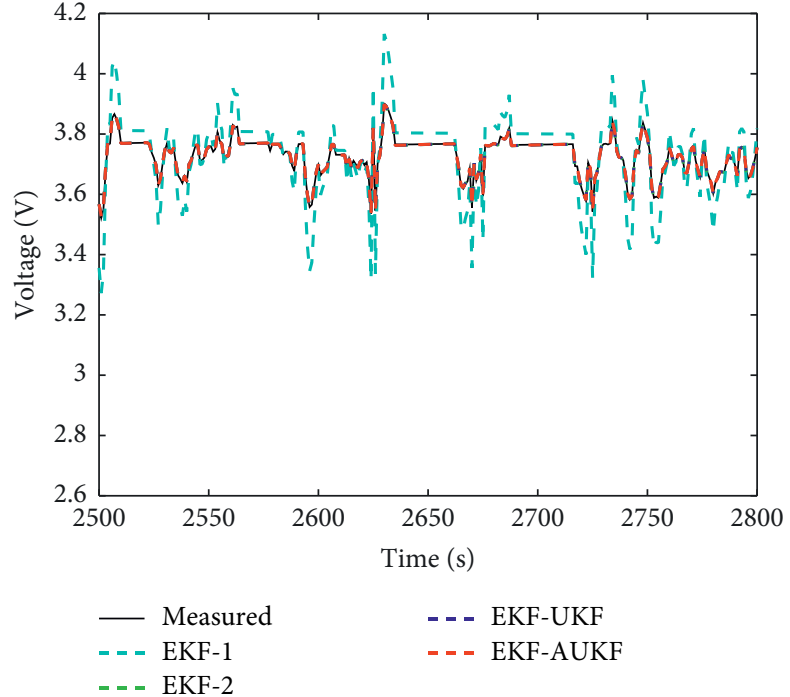

(c)

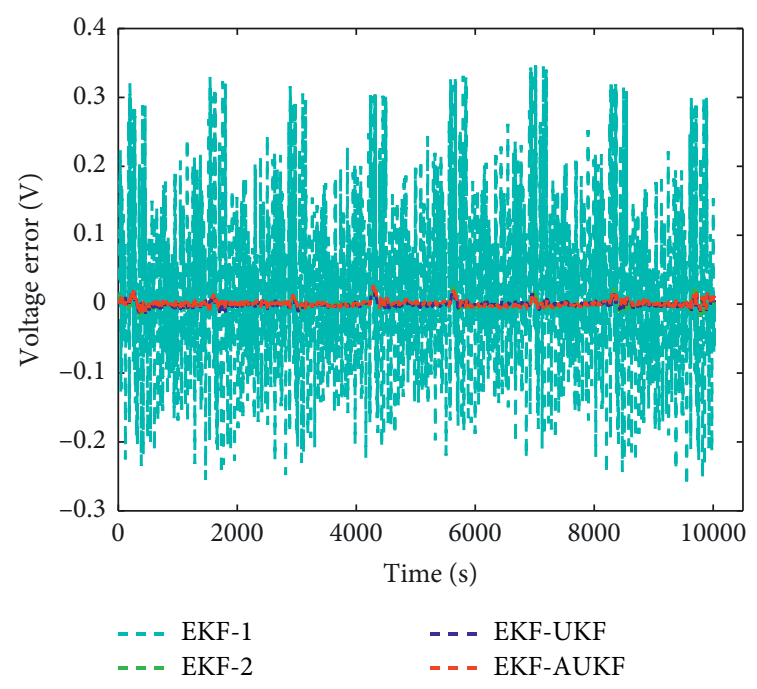

(d)

FIGURE 14: The comparison of estimation results at $45^{\circ} \mathrm{C}$ : (a) prediction results of SOC; (b) SOC error; (c) prediction results of voltage; and (d) voltage error.

TABLE 3: Error results of SOC and voltage estimation under the FUDS test.

\begin{tabular}{|c|c|c|c|c|c|}
\hline \multirow{2}{*}{$T\left({ }^{\circ} \mathrm{C}\right)$} & \multirow{2}{*}{ Method } & \multicolumn{2}{|c|}{ SOC error (\%) } & \multicolumn{2}{|c|}{ Voltage error $(\mathrm{mV})$} \\
\hline & & MAE & RMSE & MAE & RMSE \\
\hline \multirow{4}{*}{0} & EKF-1 & 5.208 & 5.70 & 32.465 & 46.135 \\
\hline & EKF-2 & 0.842 & 1.117 & 8.269 & 13.129 \\
\hline & EKF-UKF & 0.566 & 0.705 & 8.362 & 13.217 \\
\hline & EKF-AUKF & 0.371 & 0.496 & 8.758 & 13.755 \\
\hline \multirow{4}{*}{25} & EKF-1 & 13.16 & 14.078 & 119.7 & 161.18 \\
\hline & EKF-2 & 0.587 & 0.675 & 3.324 & 4.791 \\
\hline & EKF-UKF & 0.453 & 0.528 & 3.673 & 5.061 \\
\hline & EKF-AUKF & 0.364 & 0.437 & 3.002 & 4.23 \\
\hline \multirow{4}{*}{45} & EKF-1 & 6.973 & 7.391 & 67.824 & 90.608 \\
\hline & EKF-2 & 0.606 & 0.767 & 3.746 & 5.501 \\
\hline & EKF-UKF & 0.382 & 0.514 & 3.279 & 4.801 \\
\hline & EKF-AUKF & 0.318 & 0.461 & 3.145 & 4.505 \\
\hline
\end{tabular}

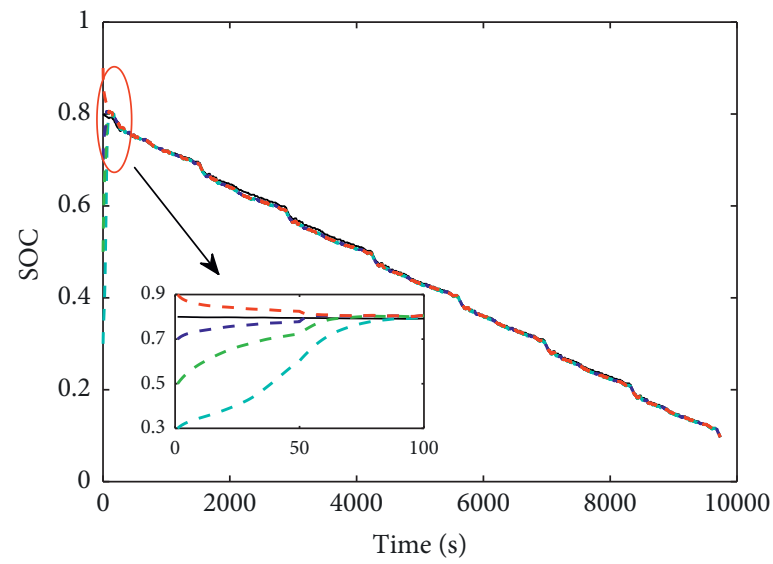

$\begin{array}{ll}\text { - } & \text { Reference } \\ \text { - - } & \text { SOC }=0.3 \\ \text { - - } & \text { SOC }=0.5\end{array}$

- - - $\mathrm{SOC}=0.7$

- - - SOC $=0.9$

(a)

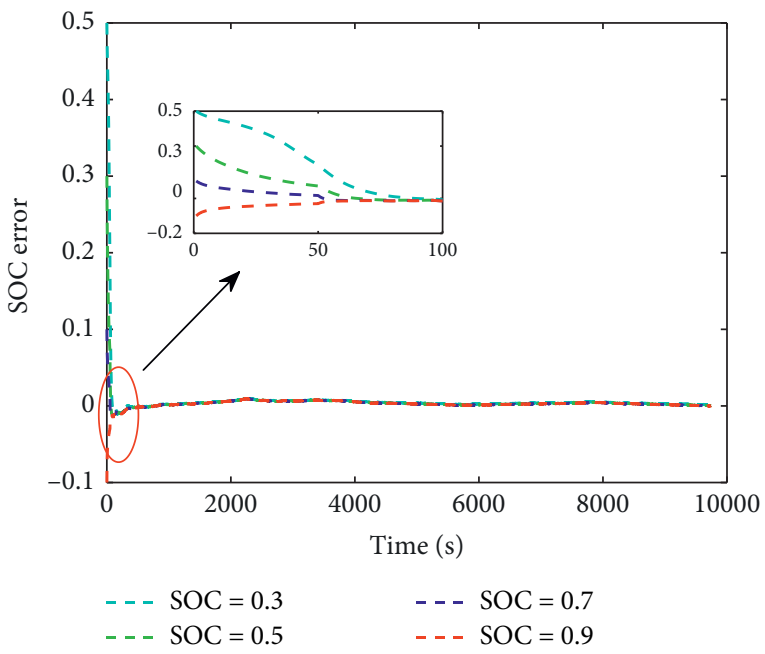

(b)

Figure 15: Continued. 


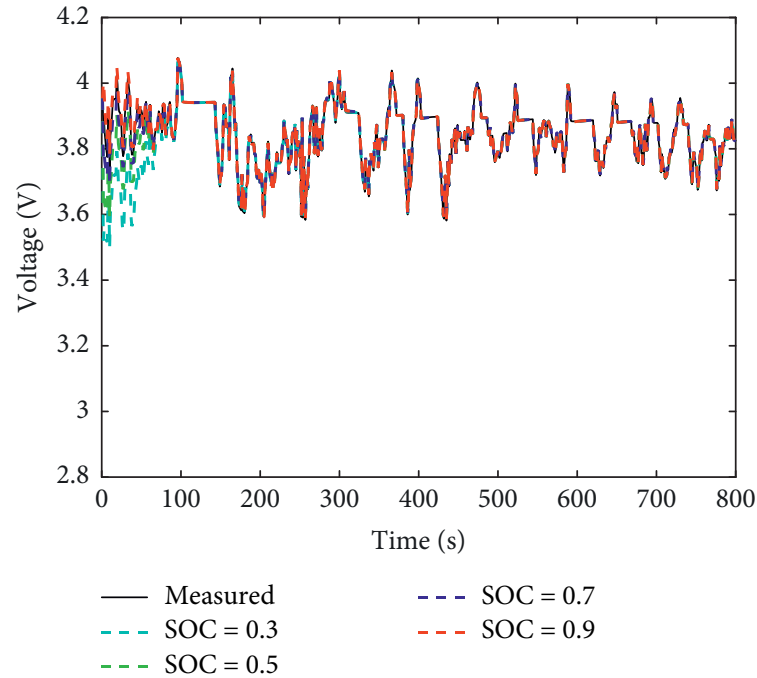

(c)

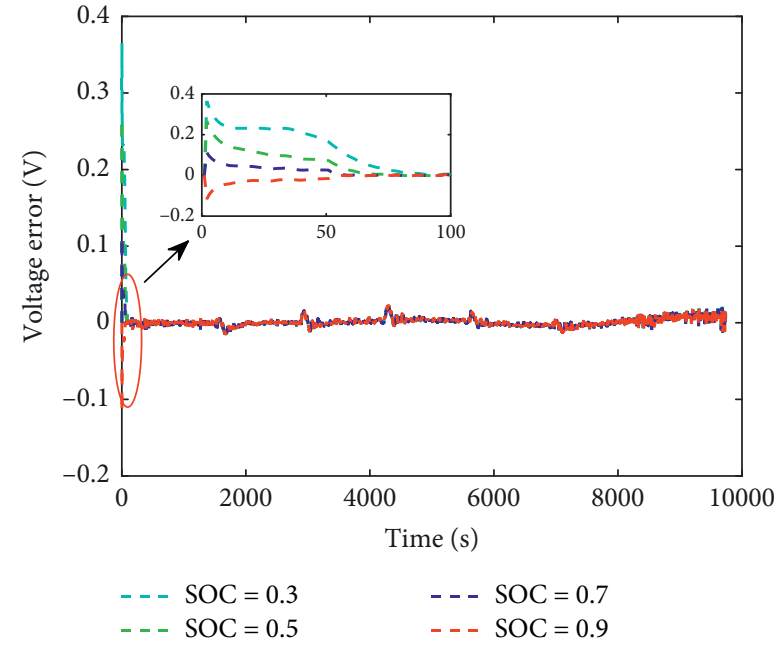

(d)

FIGURE 15: Experiment results under different initial SOC values: (a) prediction results of SOC; (b) SOC error; (c) prediction results of voltage; and (d) voltage error.

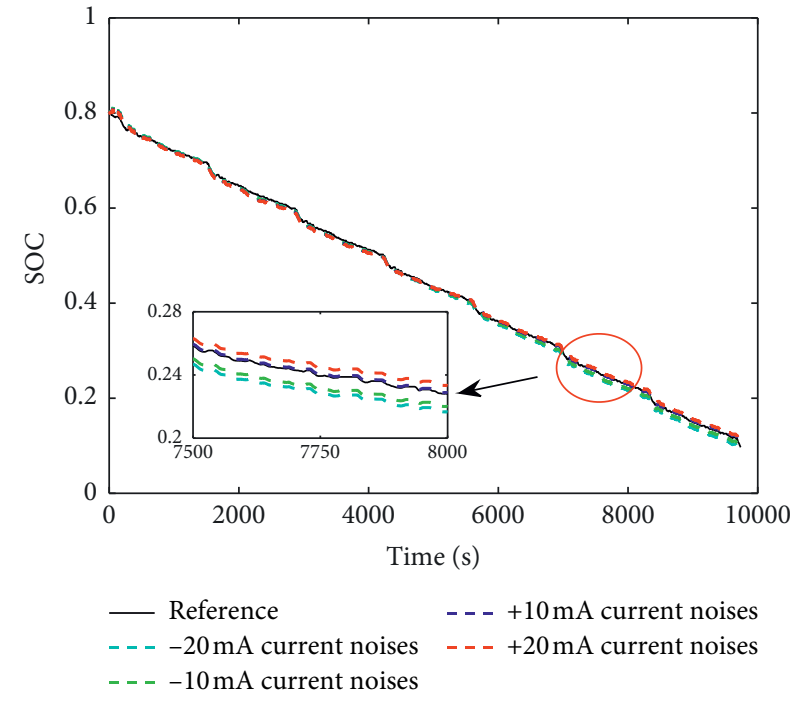

(a)

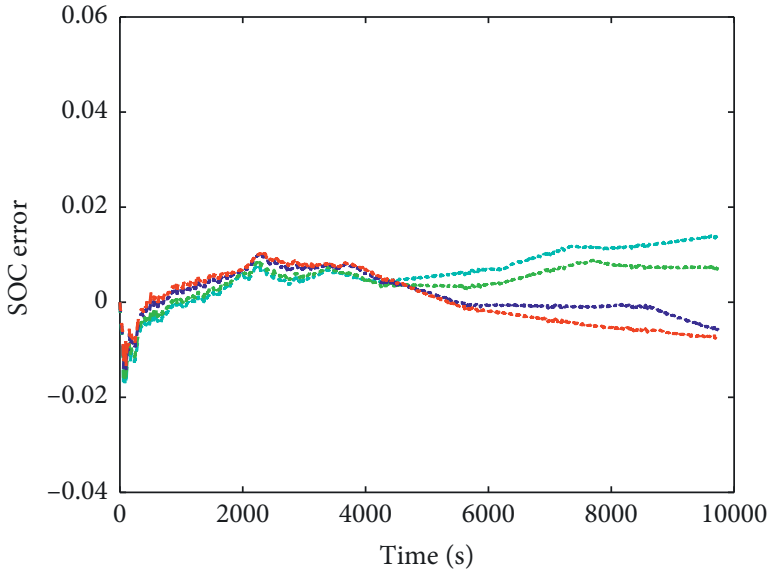

- - - $-20 \mathrm{~mA}$ current noises - - - +10 mA current noises _ _ _ - $10 \mathrm{~mA}$ current noises _ _ _ + $20 \mathrm{~mA}$ current noises

(b)

Figure 16: Continued. 


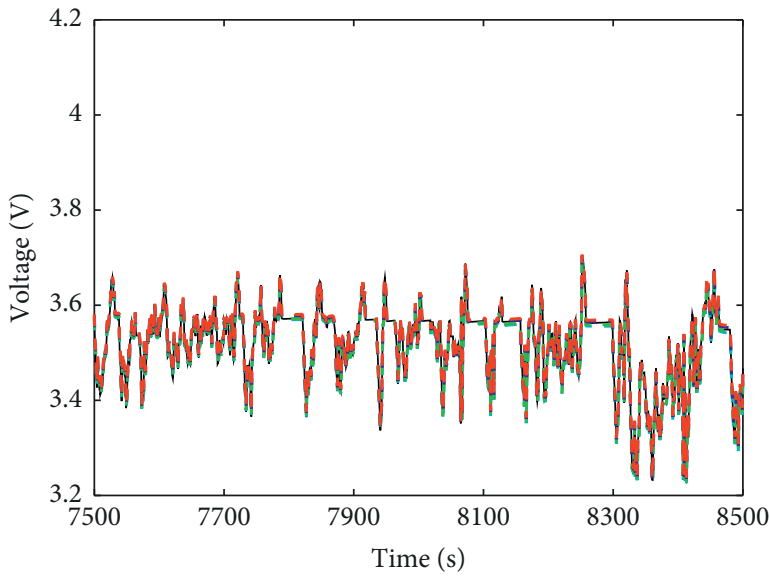

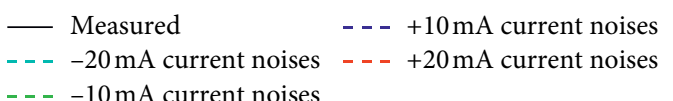

(c)

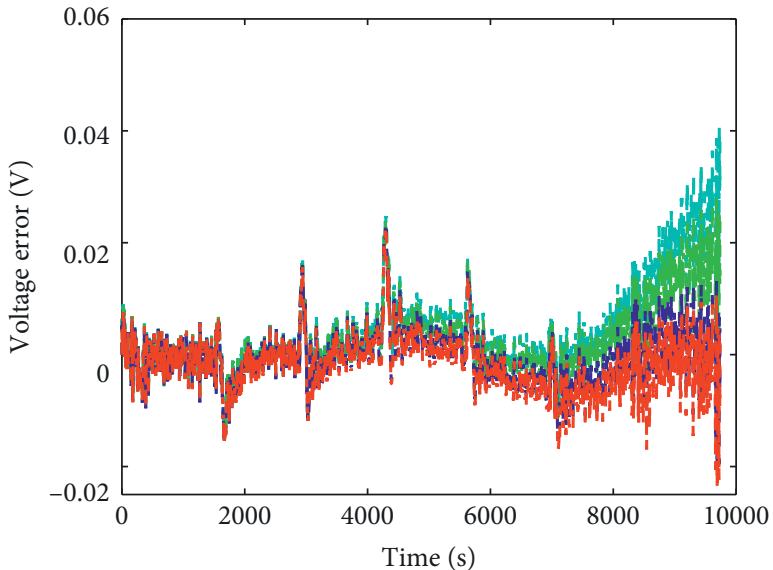

- - - $-20 \mathrm{~mA}$ current noises _ - - + $+10 \mathrm{~mA}$ current noises _ _ _ $-10 \mathrm{~mA}$ current noises _ _ _ $+20 \mathrm{~mA}$ current noises

(d)

FIGURE 16: Experimental results with current noise: (a) prediction results of SOC; (b) SOC error; (c) prediction results of voltage; and (d) voltage error.

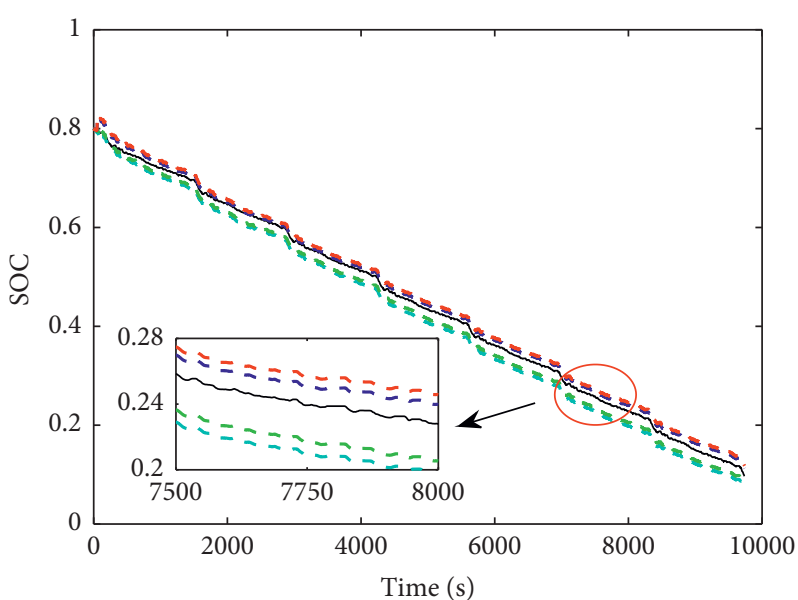

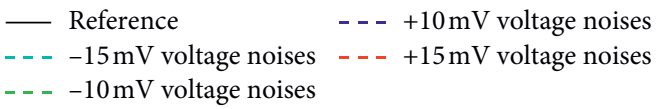

(a)

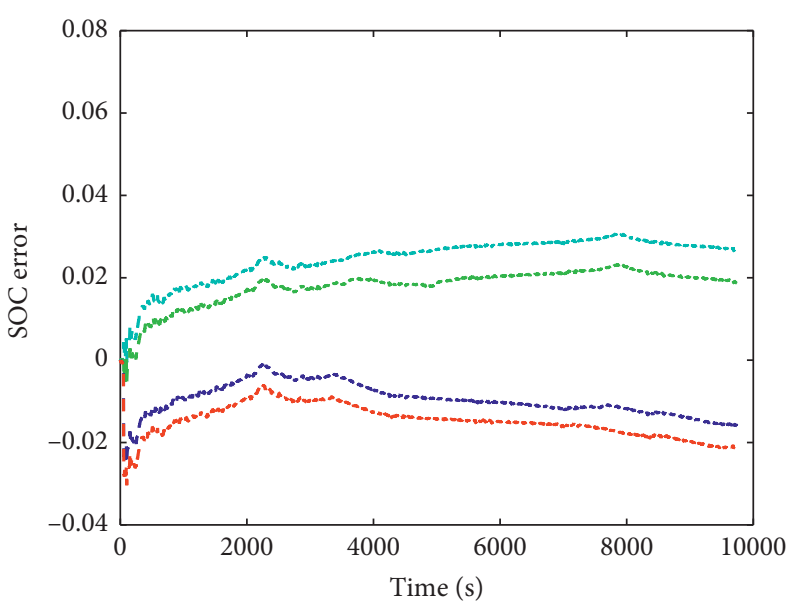

- - - -15 $\mathrm{mV}$ voltage noises - - - $+10 \mathrm{mV}$ voltage noises _ - - $-10 \mathrm{mV}$ voltage noises _ - - $+15 \mathrm{mV}$ voltage noises

FIGURE 17: Continued. 


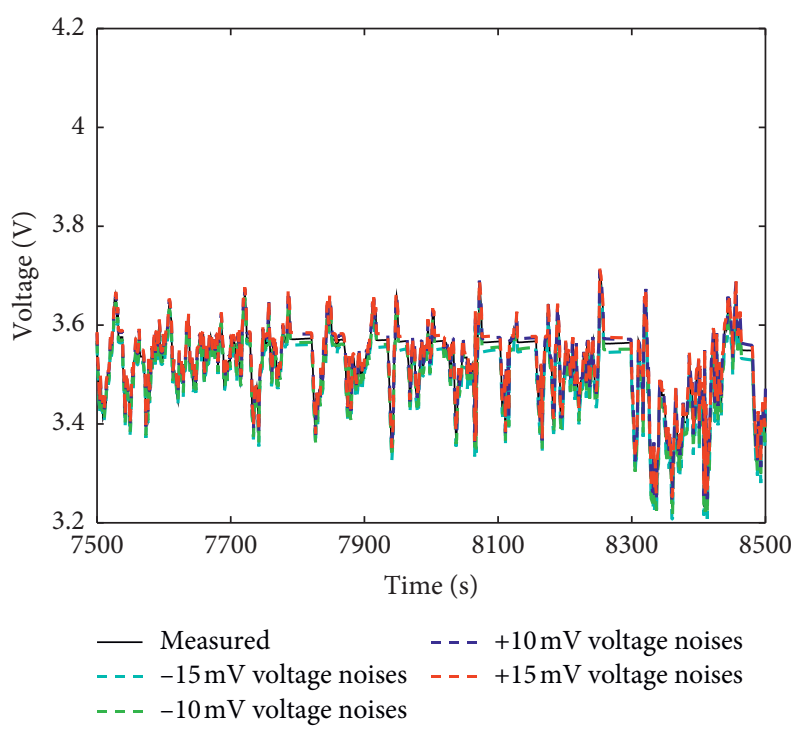

(c)

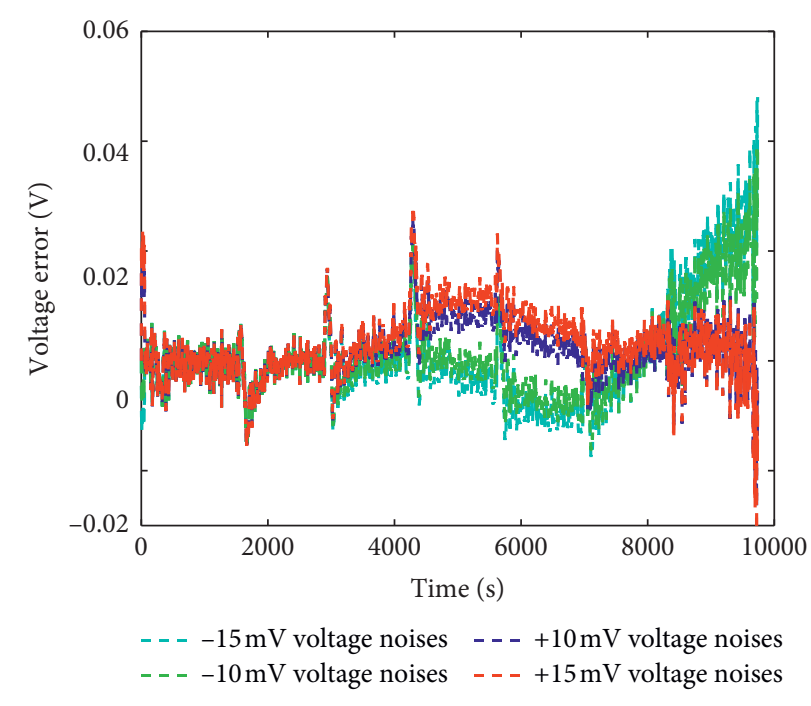

(d)

FIGURE 17: Experimental results with voltage noise: (a) prediction results of SOC; (b) SOC error; (c) prediction results of voltage; and (d) voltage error.

TABLE 4: Experimental error results with current and voltage noise.

\begin{tabular}{|c|c|c|c|c|c|}
\hline \multirow{2}{*}{ Method } & \multirow{2}{*}{ Value } & \multicolumn{2}{|c|}{ SOC error (\%) } & \multicolumn{2}{|c|}{ Voltage error $(\mathrm{mV})$} \\
\hline & & MAE & RMSE & MAE & RMSE \\
\hline \multirow{4}{*}{ Current (mA) } & -20 & 0.728 & 0.819 & 5.782 & 9.056 \\
\hline & -10 & 0.543 & 0.591 & 4.515 & 6.681 \\
\hline & +10 & 0.349 & 4.536 & 3.409 & 4.639 \\
\hline & +20 & 0.499 & 5.621 & 3.697 & 5.022 \\
\hline \multirow{4}{*}{ Voltage $(\mathrm{mV})$} & -15 & 2.457 & 2.5185 & 6.438 & 9.589 \\
\hline & -10 & 1.791 & 1.8455 & 5.327 & 7.940 \\
\hline & +10 & 0.959 & 1.0373 & 3.885 & 5.440 \\
\hline & +15 & 1.474 & 1.5283 & 4.863 & 6.622 \\
\hline
\end{tabular}

initial value. Thus, the proposed algorithm can still have strong robustness and obtain accurate SOC estimation under different SOC initial errors. The estimated values of SOC and voltage converge to the true values within $50-80 \mathrm{~s}$, indicating that the initial error of SOC has a small impact on the convergence speed of the algorithm.

\subsection{Experiment Results with Different Current and Voltage} Noises. The current and voltage of the vehicle in the actual working process are collected by the voltage and hall current sensors. The accuracy of different types of sensors is also different. Due to the influence of vehicle cost and reliability, sensors often make a trade-off between accuracy and cost. Moreover, such influences are subject to electromagnetic interference from the working environment. The current and voltage collected are inaccurate, which can eventually affect the estimation accuracy of SOC. Therefore, different noises are added to the actual measured current and voltage to test and verify the robustness of the EKF-AUKF method. The dataset used in this part is the experimental data of FUDS at $25^{\circ} \mathrm{C}$.
First, the current errors of 10 and $20 \mathrm{~mA}$ on the actual measured currents are loaded. The simulation results are shown in Figures 16(a)-16(d). Second, the voltage errors of 10 and $15 \mathrm{mV}$ on the actual measured voltages are loaded. The simulation results are shown in Figures 17(a)-17(d). Figures 16(a) and 17(a) illustrate that the SOC estimation curve can well track the actual SOC curve. Figures 16 (c) and 17 (c) illustrate that the voltage estimation curve can also track the actual measured voltage curve. The statistical error results of the experimental simulation are shown in Table 4. The RMSE of the SOC estimation under the current noise error of $20 \mathrm{~mA}$ is smaller than that of the voltage noise error of $10 \mathrm{mV}$. It indicates that the effect of the current noises on SOC estimation accuracy is smaller than that of the voltage noises. This is because the internal resistance of the battery is relatively small. When the current error occurs, this error is directly converted into the internal resistance voltage error. Its value is smaller than the error directly added to the measured voltage. It is also found that the influence of positive current or voltage error on 
estimation accuracy is less than that of the negative current or voltage error. Figures $16(\mathrm{~d})$ and $17(\mathrm{~d})$ illustrate that, at the end of battery discharge, the current and voltage errors can rapidly increase the battery terminal voltage error, which will eventually reduce the prediction accuracy of SOC. Therefore, in practical applications, the accuracy of the voltage sensor should be ensured as much as possible, and the excessive discharge of the battery must be avoided.

\section{Conclusions}

In this paper, three methods of model parameter identification are analyzed and discussed in detail. Aiming at the shortcomings of traditional parameter identification methods, a double Kalman filter is used to update the parameters and SOC of the battery online. At the same time, the self-adaptive noise method is used to improve the predictive performance of the conventional UKF algorithm. That is, EKF is used to update the model parameters at the macroscale, and then the AUKF is applied to estimate the SOC of the battery on the microscale.

The offline parameter identification of the first-order RC model was performed using the ordinary RLS method under the HPPC condition. Simulation verification was then conducted on the model. The simulated voltage errors at three temperatures are within $0.01 \mathrm{~V}$. The results show that the identified parameters of the three temperatures can meet the simulation requirements in HPPC condition. The FFRLS algorithm is used to identify the model parameters under the FUDS condition. Then, the EKF algorithm was used to estimate the SOC and voltage of the battery models identified by the two methods. The results reveal that the SOC and voltage errors of the EKF2 algorithm are smaller than the simulation errors of the EKF-1 algorithm, indicating that the offline parameters identified by the HPPC condition are inapplicable to the FUDS condition. In the entire FUDS test, the three other algorithms can obtain accurate SOC values at different temperatures, and the RMSE of SOC is less than $2 \%$. However, the EKF-UKF and EKF-AUKF can obtain more accurate $S O C$ values by updating the model parameters online than the EKF with fixed parameters. The EKFAUKF algorithm with adaptive noise has a more accurate SOC value than the EKF-UKF algorithm. The predicted SOC and voltage curves converge quickly to the true value within 50-80 s under different initial SOC errors; the RMSE of SOC is less than $1 \%$, and the RMSE of voltage is within $0.01 \mathrm{~V}$. The result shows that the proposed algorithm can obtain accurate SOC values and has strong robustness. The effects of current and voltage noise errors on SOC and voltage simulations are analyzed and discussed. The experimental results reveal that the effect of current noise error on the accuracy of SOC estimation is less than that of voltage noise error. The algorithm can meet the requirements of working conditions within a voltage error of $10 \mathrm{mV}$ or a current error of $20 \mathrm{~mA}$.
Due to the limited sample size of the dataset, this study does not discuss the problem that the identification parameters of HPPC condition cannot be applied to the FUDS working condition. This limitation may be due to the battery charge and discharge rate. The influences of charge and discharge ratios on model parameter identification can be investigated in future studies.

\section{Data Availability}

The data used to support the findings of this study have been uploaded to the website (https://web.calce.umd.edu/ batteries/data.htm\#type3) which can be downloaded freely.

\section{Conflicts of Interest}

The authors declare that they have no conflicts of interest.

\section{Acknowledgments}

This research was funded by the Key Tackling Item in Science and Technology Department of Jilin Province, China, under Grant number 20150204017GX, and Jilin Provincial Natural Science Foundation, China, under Grant number 20150101037JC.

\section{References}

[1] M. A. Hannan, M. S. H. Lipu, A. Hussain, and A. Mohamed, "A review of lithium-ion battery state of charge estimation and management system in electric vehicle applications: challenges and recommendations," Renewable and Sustainable Energy Reviews, vol. 78, pp. 834-854, 2017.

[2] L. H. Saw, Y. Ye, and A. A. O. Tay, "Integration issues of lithium-ion battery into electric vehicles battery pack," Journal of Cleaner Production, vol. 113, pp. 1032-1045, 2016.

[3] C. Zhang, Y. He, B. Du, L. Yuan, B. Li, and S. Jiang, "Transformer fault diagnosis method using IoT based monitoring system and ensemble machine learning," Future Generation Computer Systems, vol. 108, pp. 533-545, 2020.

[4] C. Zhang, Y. He, L. Yuan, and S. Xiang, "Capacity prognostics of lithium-ion batteries using EMD denoising and multiple kernel RVM,” IEEE Access, vol. 5, pp. 12061-12070, 2017.

[5] B. Xia, H. Wang, Y. Tian, M. Wang, W. Sun, and Z. Xu, "State of charge estimation of lithium-ion batteries using an adaptive cubature Kalman filter," Energies, vol. 8, no. 6, pp. 5916-5936, 2015.

[6] N. Yang, X. Zhang, and G. Li, "State of charge estimation for pulse discharge of a $\mathrm{LiFePO}_{4}$ battery by a revised $\mathrm{Ah}$ counting," Electrochimica Acta, vol. 151, pp. 63-71, 2015.

[7] S. Lee, J. Kim, J. Lee, and B. H. Cho, "State-of-charge and capacity estimation of lithium-ion battery using a new opencircuit voltage versus state-of-charge," Journal of Power Sources, vol. 185, no. 2, pp. 1367-1373, 2008.

[8] L. Chen, Z. Wang, Z. Lu et al., "A novel state-of-charge estimation method of lithium-ion batteries combining the grey model and genetic algorithms," IEEE Transactions on Power Electronics, vol. 33, no. 10, pp. 8797-8807, 2018.

[9] M. Luzi, M. Paschero, A. Rizzi, E. Maiorino, and F. M. Frattale Mascioli, "A novel neural networks ensemble approach for modeling electrochemical cells," IEEE Transactions on Neural 
Networks and Learning Systems, vol. 30, no. 2, pp. 343-354, 2019.

[10] G. Dong, X. Zhang, C. Zhang, and Z. Chen, "A method for state of energy estimation of lithium-ion batteries based on neural network model," Energy, vol. 90, pp. 879-888, 2015.

[11] J. Meng, G. Luo, and F. Gao, "Lithium polymer battery stateof-charge estimation based on adaptive unscented kalman filter and support vector machine," IEEE Transactions on Power Electronics, vol. 31, no. 3, pp. 2226-2238, 2016.

[12] E. Chemali, P. J. Kollmeyer, M. Preindl, R. Ahmed, and A. Emadi, "Long short-term memory networks for accurate state-of-charge estimation of Li-ion batteries," IEEE Transactions on Industrial Electronics, vol. 65, no. 8, pp. 6730-6739, 2018.

[13] F. Yang, W. Li, C. Li, and Q. Miao, "State-of-charge estimation of lithium-ion batteries based on gated recurrent neural network," Energy, vol. 175, pp. 66-75, 2019.

[14] R. Xiong, X. Gong, C. C. Mi, and F. Sun, "A robust state-ofcharge estimator for multiple types of lithium-ion batteries using adaptive extended Kalman filter," Journal of Power Sources, vol. 243, pp. 805-816, 2013.

[15] X. Guo, L. Kang, Y. Yao, Z. Huang, and W. Li, "Joint estimation of the electric vehicle power battery state of charge based on the least squares method and the kalman filter algorithm," Energies, vol. 9, no. 2, p. 100, 2016.

[16] Z. Wei, S. Meng, B. Xiong, D. Ji, and K. J. Tseng, "Enhanced online model identification and state of charge estimation for lithium-ion battery with a FBCRLS based observer," Applied Energy, vol. 181, pp. 332-341, 2016.

[17] C. Hu, B. D. Youn, and J. Chung, "A multiscale framework with extended Kalman filter for lithium-ion battery SOC and capacity estimation,” Applied Energy, vol. 92, pp. 694-704, 2012.

[18] X. Zhang, Y. Wang, D. Yang, and Z. Chen, "An on-line estimation of battery pack parameters and state-of-charge using dual filters based on pack model," Energy, vol. 115, pp. 219229, 2016.

[19] G. Dong, Z. Chen, J. Wei, C. Zhang, and P. Wang, "An online model-based method for state of energy estimation of lithiumion batteries using dual filters," Journal of Power Sources, vol. 301, pp. 277-286, 2016.

[20] C. Chen, R. Xiong, and W. Shen, "A lithium-ion battery-inthe-loop approach to test and validate multiscale dual $\mathrm{H}$ infinity filters for state-of-charge and capacity estimation," IEEE Transactions on Power Electronics, vol. 33, no. 1, pp. 332-342, 2018.

[21] R. Xiong, Y. Zhang, H. He, X. Zhou, and M. G. Pecht, "A double-scale, particle-filtering, energy state prediction algorithm for lithium-ion batteries," IEEE Transactions on Industrial Electronics, vol. 65, no. 2, pp. 1526-1538, 2018.

[22] Y. Wang, C. Zhang, and Z. Chen, "An adaptive remaining energy prediction approach for lithium-ion batteries in electric vehicle," Journal of Power Sources, vol. 305, pp. 80-88, 2016.

[23] B. Xia, Z. Sun, R. Zhang et al., "A comparative study of three improved algorithms based on particle filter algorithms in SOC estimation of lithium ion batteries," Energies, vol. 10, no. 8, p. 1149, 2017.

[24] X. Liu, Z. Chen, C. Zhang, and J. Wu, "A novel temperaturecompensated model for power Li-ion batteries with dualparticle-filter state of charge estimation," Applied Energy, vol. 123, pp. 263-272, 2014.

[25] Q. Yu, R. Xiong, C. Lin, W. Shen, and J. Deng, "Lithium-ion battery parameters and state-of-charge joint estimation based on H-infinity and unscented kalman filters," IEEE Transactions on Vehicular Technology, vol. 66, no. 10, pp. 8693-8701, 2017.
[26] X. Han, M. Ouyang, L. Lu, and J. Li, "Simplification of physics-based electrochemical model for lithium ion battery on electric vehicle. Part II: pseudo-two-dimensional model simplification and state of charge estimation," Journal of Power Sources, vol. 278, pp. 814-825, 2015.

[27] M. A. Rahman, S. Anwar, and A. Izadian, "Electrochemical model parameter identification of a lithium-ion battery using particle swarm optimization method," Journal of Power Sources, vol. 307, pp. 86-97, 2016.

[28] X. Hu, S. Li, and H. Peng, "A comparative study of equivalent circuit models for Li-ion batteries," Journal of Power Sources, vol. 198, pp. 359-367, 2012.

[29] X. Lai, Y. Zheng, and T. Sun, "A comparative study of different equivalent circuit models for estimating state-of-charge of lithium-ion batteries," Electrochimica Acta, vol. 259, pp. 566-577, 2018.

[30] Y. Tian, D. Li, J. Tian, and B. Xia, "State of charge estimation of lithium-ion batteries using an optimal adaptive gain nonlinear observer," Electrochimica Acta, vol. 225, pp. 225-234, 2017.

[31] B. Xia, Z. Lao, R. Zhang et al., "Online parameter identification and state of charge estimation of lithium-ion batteries based on forgetting factor recursive least squares and nonlinear Kalman filter," Energies, vol. 11, no. 1, p. 3, 2018.

[32] M. J. Rothenberger, D. J. Docimo, M. Ghanaatpishe, and H. K. Fathy, "Genetic optimization and experimental validation of a test cycle that maximizes parameter identifiability for a Li-ion equivalent-circuit battery model," Journal of Energy Storage, vol. 4, pp. 156-166, 2015.

[33] T. Mesbahi, F. Khenfri, N. Rizoug, K. Chaaban, P. Bartholomeüs, and P. Le Moigne, "Dynamical modeling of Li-ion batteries for electric vehicle applications based on hybrid Particle SwarmNelder-Mead (PSO-NM) optimization algorithm," Electric Power Systems Research, vol. 131, pp. 195-204, 2016.

[34] K. Zhang, J. Ma, X. Zhao, X. Liu, and Y. Zhang, "Parameter identification and state of charge estimation of NMC cells based on improved ant lion optimizer," Mathematical Problems in Engineering, vol. 2019, Article ID 4961045, 18 pages, 2019.

[35] T. Huria, M. Ceraolo, J. Gazzarri, and R. Jackey, "High fidelity electrical model with thermal dependence for characterization and simulation of high power lithium battery cells," in Proceedings of the 2012 IEEE International Electric Vehicle Conference, pp. 1-8, IEEE, Greenville, SC, USA, March 2012.

[36] J. Gazzarri, N. Shrivastava, R. Jackey, and C. Borghesani, "Battery pack modeling, simulation, and deployment on a multicore real time target," SAE International Journal of Aerospace, vol. 7, no. 2, pp. 207-213, 2014.

[37] F. Yang, Y. Xing, D. Wang, and K.-L. Tsui, “A comparative study of three model-based algorithms for estimating state-ofcharge of lithium-ion batteries under a new combined dynamic loading profile," Applied Energy, vol. 164, pp. 387-399, 2016.

[38] F. Zheng, Y. Xing, J. Jiang, B. Sun, J. Kim, and M. Pecht, "Influence of different open circuit voltage tests on state of charge online estimation for lithium-ion batteries," Applied Energy, vol. 183, pp. 513-525, 2016.

[39] Q. Zhu, M. Xu, W. Liu, and M. Zheng, "A state of charge estimation method for lithium-ion batteries based on fractional order adaptive extended Kalman filter," Energy, vol. 187, p. 115880, 2019.

[40] Y. Xing, W. He, M. Pecht, and K. L. Tsui, "State of charge estimation of lithium-ion batteries using the open-circuit voltage at various ambient temperatures," Applied Energy, vol. 113, pp. 106-115, 2014. 